\title{
The Self\#Regulated Growth of Supermassive Black Holes
}

\section{Citation}

Younger, Joshua D., Philip F. Hopkins, T. J. Cox, and Lars Hernquist. 2008. “The Self\#Regulated Growth of Supermassive Black Holes." The Astrophysical Journal 686 (2): 815-28. https:// doi.org/10.1086/591639.

\section{Permanent link}

http://nrs.harvard.edu/urn-3:HUL.InstRepos:41381777

\section{Terms of Use}

This article was downloaded from Harvard University's DASH repository, and is made available under the terms and conditions applicable to Open Access Policy Articles, as set forth at http:// nrs.harvard.edu/urn-3:HUL.InstRepos:dash.current.terms-of-use\#OAP

\section{Share Your Story}

The Harvard community has made this article openly available.

Please share how this access benefits you. Submit a story.

Accessibility 
Draft VERSiOn OCTOBER 25, 2018

Preprint typeset using $\mathrm{L}_{\mathrm{T}} \mathrm{E} \mathrm{X}$ style emulateapj v. 08/22/09

\title{
THE SELF-REGULATED GROWTH OF SUPERMASSIVE BLACK HOLES
}

\author{
Joshua D. Younger ${ }^{1,2}$, Philip F. Hopkins ${ }^{1}$, T. J. Cox ${ }^{1,3}$, \& Lars Hernquist ${ }^{1}$ \\ Draft version October 25, 2018
}

\begin{abstract}
We present a series of simulations of the self-regulated growth of supermassive black holes (SMBHs) in galaxies via three different fueling mechanisms: major mergers, minor mergers, and disk instabilities. The SMBHs in all three scenarios follow the same black hole fundamental plane (BHFP) and correlation with bulge binding energy seen in simulations of major mergers, and observed locally. Furthermore, provided that the total gas supply is significantly larger than the mass of the SMBH, its limiting mass is not influenced by the amount of gas available or the efficiency of black hole growth. This supports the assertion that SMBHs accrete until they reach a critical mass at which feedback is sufficient to unbind the gas locally, terminating the inflow and stalling further growth. At the same time, while minor and major mergers follow the same projected correlations (e.g., the $M_{B H}-\sigma$ and Magorrian relations), SMBHs grown via disk instabilities do not, owing to structural differences between the host bulges. This finding is supported by recent observations of SMBHs in pseudobulges and bulges in barred systems, as compared to those hosted by classical bulges. Taken together, this provides support for the BHFP and binding energy correlations as being more "fundamental" than other proposed correlations in that they reflect the physical mechanism driving the co-evolution of SMBHs and spheroids.
\end{abstract}

Subject headings: galaxies: formation - galaxies: evolution - black hole physics - galaxies: general methods: numerical

\section{INTRODUCTION}

Over the past two decades, it has been established that supermassive black holes (SMBHs) in the nuclei of galaxies are common, and that their masses are connected to the properties of their hosts. As the sample of robust SMBH measurements has increased (Kormendy \& Richstone 1995; Kormendy 2004) several black hole - host galaxy correlations (see Novak et al. 2006, for a review) have been proposed, including central velocity dispersion (Ferrarese \& Merritt 2000; Gebhardt et al. 2000a; Tremaine et al. 2002), bulge mass/luminosity (Kormendy \& Richstone 1995; Magorrian et al. 1998; McLure \& Dunlop 2002; Marconi \& Hunt 2003; Häring \& Rix 2004), light concentration (Graham et al. 2001; Graham \& Driver 2007), and binding energy (Aller \& Richstone 2007). Recently, it has been suggested that these observed correlations may be projections of a "Black-Hole Fundamental Plane" (BHFP: Hopkins et al. 2007a,b; Aller \& Richstone 2007; Barway \& Kembhavi 2007), analogous to that describing the structural properties of elliptical galaxies (Dressler et al. 1987; Diorgovski \& Davis 1987), that is manifest as a tilted bulge binding energy correlation. These relationships are indicative of an intimate connection between SMBH growth and galaxy formation/evolution.

There is also increasing evidence, both direct and indirect, that the growth of SMBHs is self-regulated, with strong feedback from the active galactic nucleus (AGN) playing an important role. Numerical simulations of major mergers of gas-rich spirals that include this

\footnotetext{
${ }^{1}$ Harvard-Smithsonian Center for Astrophysics, 60 Garden Street, Cambridge, MA 02138

2 jyounger@cfa.harvard.edu

3 Keck Foundation Fellow
}

process, along with gas dissipation, naturally account for the observed SMBH correlations (Di Matteo et al. 2005; Robertson et al. 2006c; Hopkins et al. 2007a; Johansson et al. 2008), the fundamental plane of elliptical galaxies (Robertson et al. 2006b; Hopkins et al. 2008h), the structure (Hopkins et al. 2008c f, e) and stellar kinematics (Cox et al. 2006) of massive ellipticals, and the redshift evolution of the correlations (Hopkins et al. 2007a, 2008g). Moreover, a strong feedback mode has proven essential in semi-analytic models and numerical simulations of hierarchical galaxy formation (e.g., Croton et al. 2006; Bower et al. 2006; Sijacki et al. 2007; Di Matteo et al. 2008, Sommerville et al. 2008, in prep.) and the merger-driven evolution of quasars and red galaxies (Hopkins et al. 2005a, b d, 2006a, c, 2008a, d). The prevalence of this process is further supported by direct observational evidence for outflows in both Seyfert galaxies (e.g., Crenshaw et al. 1999; Kriss et al. 2000; Kaastra et al. 2000, 2002; Kaspi et al.|2000a, 2002) and more luminous quasars (e.g., Weymann et al. 1991; Korista et al. 1993; Arav et al. 2001) driven by strong thermal and radiative feedback originating from the AGN (see review by Crenshaw et al. 2003).

Despite these observational and theoretical advances, the fundamental character of these SMBH correlations remains poorly understood. It appears clear the SMBHs are closely linked with the structural properties of their host galaxy's bulge (see e.g., Novak et al. 2006). However, an important question has not been adequately addressed: to the extent that the growth of SMBHs is selfregulated, what property - or properties - of this bulge does the SMBH "see?" The more "fundamental" the scaling relation, the more it reflects the physical mechanism driving the co-evolution of SMBHs and bulges. Hopkins et al. (2007a) suggest that the final mass of the 

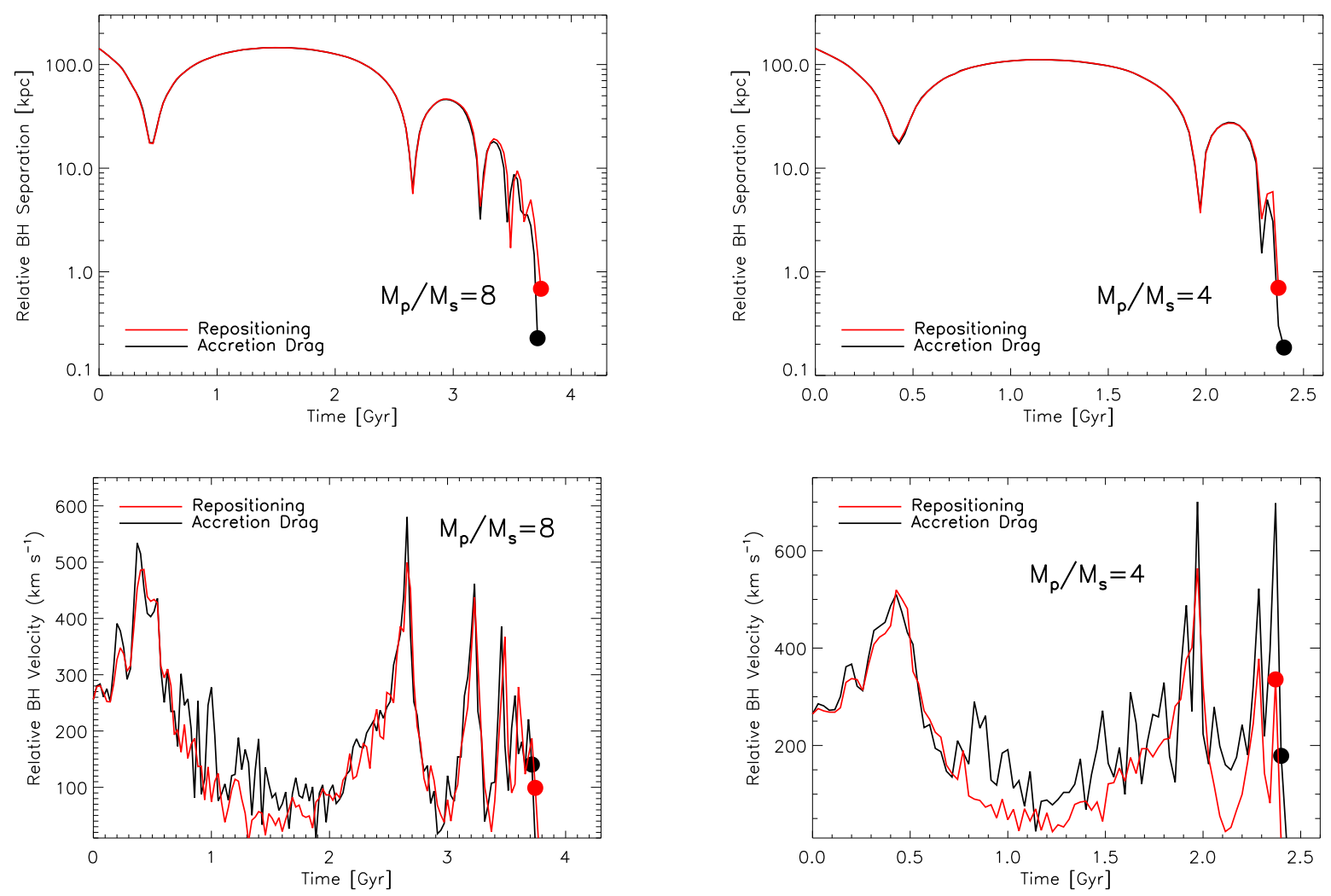

FIG. 1. - Top: Relative separation of the two SMBHs over the course of an $M_{p} / M_{s}=8$ (left) and 4 (right) merger simulation. Included are results using both the accretion drag (black line; see $\S 2.3$ for details) and repositioning (red line; see Johansson et al. 2008) methods. The circles indicate the time of the merger. Botтom: Same labeling for the relative velocities of the two SMBHs over the course of the simulation.

SMBH is set by the depth of the local potential; the SMBH grows until feedback unbinds the local gas supply, abruptly terminating its growth. However, this hypothesis, while promising, has not yet been systematically tested.

In this paper, we use hydrodynamical simulations to examine the self-regulated growth of SMBHs in three different fueling modes: major mergers, minor mergers, and disk instabilities. Our aim is to investigate the physical mechanism that determines the final mass of the $\mathrm{SMBH}$. It is organized as follows: in $\S 2$ we review our methodology, in $\S 3$ we describe the simulations, in $\S 4$ we present our results, and in $\S 5$ we discuss implications.

\section{METHODS}

\subsection{Hydrodynamics}

In what follows, we describe a series of simulations run using PGADGET2 (Springel 2005), a massively parallel smoothed particle hydrodynamics code (SPH: see also Lucv 1977; Gingold \& Monaghan 1977) using the entropy conserving formulation of Springel \& Hernquist (2002), coupled with a Tree algorithm (Barnes \& Hut 1986) to compute the gravitational forces between particles (see also Hernquist \& Katz 1989). We also include the effects of radiative cooling (see Katz et al. 1996), and employ a sub-resolution model for the interstellar medium (ISM) to describe star formation and supernova feedback, tuned to reproduce the local Schmidt Law (Schmidt 1959; Kennicutt 1998), that pressurizes the star-forming gas via an effective equation of state $q_{E O S}$ that interpolates between an isothermal $\left(q_{E O S}=0\right)$ and full multiphase ISM $\left(q_{E O S}=1\right.$; Springel \& Hernquist 2003). This sub-resolution model is essential for simulations of gas rich systems, because it allows us to evolve disks of arbitrary gas content without fragmenting owing to Toomre (1964) instability (see Springel \& Hernquist 2003, 2005; Robertson et al. 2004, 2006a).

\section{2. $S M B H$ Growth and Feedback}

SMBHs in our simulations are represented by "sink" particles, which increase their mass at the rate $\dot{M}_{B H}$ via Eddington-limited Bondi-Hoyle-Lyttleton accretion (Hovle \& Lyttleton 1941; Bondi \& Hoyle 1944; Bondi 1952) of the surrounding gas. As the numerical prefactor for the accretion rate - Bondi accretion is a dimensionless scaling - we adopt an efficiency of $\alpha=100$ for all the simulations used in this work (see also Springel et al. 2005; Johansson et al. 2008). However, this choice matters little as the SMBH's growth is Eddington limited during all the times of interest. The bolometric luminosity of the $\mathrm{SMBH}$ is assumed to be $L_{b o l}=\epsilon \dot{M} c^{2}$, where $\epsilon=0.1$ is the canonical radiative efficiency for thin-disk accretion. Feedback is implemented by coupling a fixed fraction of this luminosity (feedback efficiency $\eta$; typically $\eta \approx 5 \%$ ) to its environment by injecting thermal energy into the surrounding gas, weighted by the SPH smoothing kernel. The net ratio between the dimensional accretion rate (Bondi or Eddington) and the thermal feedback input into the gas is in some sense the only free parameter $-\alpha, \epsilon$, and $\eta$ are degenerate - in our feedback model, 

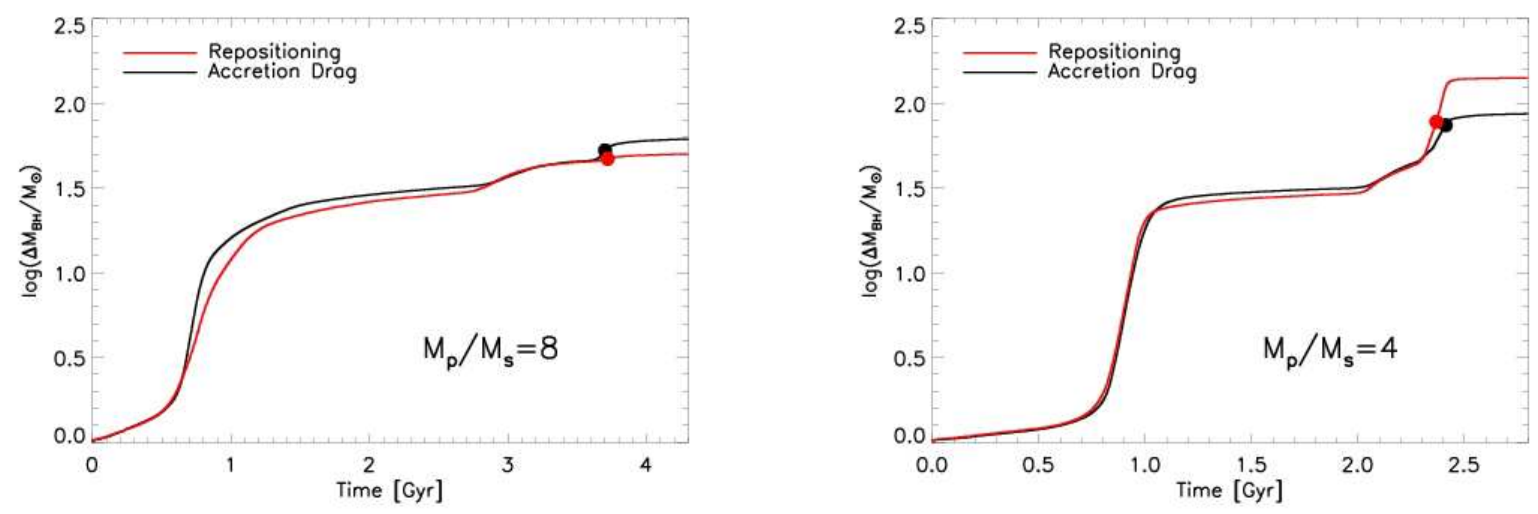

Fig. 2.- Growth of the SMBH over the course of an $M_{p} / M_{s}=8$ (left) and 4 (right) merger simulation. Included are results using both the accretion drag (black line; see $\S 2.3$ for details) and repositioning (red line; see Johansson et al. 2008) methods. The circles indicate the time of the merger. There is no systematic trend with the choice of either the accretion drag or repositioning methods.

and that ratio is chosen to reproduce the normalization of the local $M_{B H}-\sigma$ relation (Di Matteo et al. 2005; Robertson et al. 2006c); the slopes and relative normalizations of the resulting correlations of SMBH mass with galaxy properties are generic, and are generally not tunable.

It may be argued that this specific method of implementing feedback - in this case an isotropic thermal coupling - is significant for our results, especially in the absence of constraints from a well-understood, detailed model for AGN feedback. It is worth noting that alternate feedback prescriptions have led to similar results as those presented here (e.g., Murray et al. 2005). However, more generally our isotropic thermal coupling to the surrounding gas is a good approximation to a variety of feedback mechanisms, as it leads to a shock front which isotropizes and becomes wellmixed over physical scales smaller than those relevant to our simulations, and in timescales smaller than the dynamical time of the galaxy (Hopkins \& Hernquist 2006; Hopkins et al. 2006b). ${ }^{4}$ The spatial (i.e., SPH smoothing length) resolution for the simulations analyzed here is $30-50 \mathrm{pc}$, and the dynamical time over those scales is $t_{d y n}(30-50 \mathrm{pc}) \sim 10^{7}$ years. Numerical experiments have shown that shock fronts, even if initially highly beamed, will become spherical on an isotropization timescale of $t_{\text {ISO }} \approx 6 \times 10^{3}\left(E_{51} / \rho_{24}\right)^{1 / 3}$ years - where $E_{51}$ is the input energy in units of $10^{51}$ ergs and $\rho_{24}$ is the density of the ambient ISM in units of $10^{-24} \mathrm{~g} \mathrm{~cm}^{-3}$ and size scales of $\lesssim 10$ pc (Aval \& Piran 2001), both of which are below scales probed by our simulations. Therefore, for the simulations presented here, we believe that an isotropic thermal coupling prescription for feedback offers a good approximation (for further discussion, see Hopkins et al. 2006a); an assertion that we will test in detail in due course.

\subsection{Black Hole Mergers}

4 This approximation is not valid in the case of "radio-mode" feedback (Croton et al. 2006), in which MHD effects can maintain a collimated jet on scale comparable to the galaxy. However, observations suggest that black holes gain most of their mass in bright quasar modes (Soltan 1982), and $\lesssim 10 \%$ of these objects are radioloud. Therefore, we do not believe that this will be the relevant feedback mechanism for the growth mechanisms presented here.
In simulations of interacting systems the implementation of SMBH mergers can potentially introduce a systematic bias in the final SMBH mass. Absent resolution constraints, we would expect the $\mathrm{BH}$ binary to eventually harden via a combination of dynamical (Makino \& Funato 2004; Berczik et al. 2006) and hydrodynamic (Escala et al. 2004) processes, at which point gravitational wave emission dominates the energy loss and the two SMBHs in-spiral and coalesce (e.g., Milosavljević \& Merritt 2001). However, because computational limitations prevent following this evolution in a galaxy-scale simulation - and gravitational radiation is not modeled at all - the standard prescription adopted by Springel et al. (2005) merges the two SMBHs if they are within a smoothing length with a relative velocity less than or equal to the local sound speed.

Like Johansson et al. (2008), we found that this simple criterion was not in of itself adequate to ensure rapid merging of the SMBHs at the end of the merger - particularly for the unequal mass interactions. However, rather than employ their repositioning scheme - wherein the $\mathrm{SMBH}$ is repositioned at every timestep at the minimum of the local potential - we introduced a term accounting for Bondi accretion drag (Edgar 2004):

$$
F_{d r a g}=\dot{M}_{B H} v_{\infty}
$$

where $v_{\infty}$ is the initial infall speed of the gas in the Bondi approximation. This is a very approximate treatment (for a more detailed analysis, see Ruderman \& Spiegel 1971), but captures the essential physics at a level that is adequate for our purposes.

To investigate the sensitivity of our results to this choice of numerical implementation, we ran two representative simulations - an $M_{p} / M_{s}=8$ and 4 interaction with initial gas fractions of $f_{g}=0.8^{5}$ and 0.4 respectively and identical orbital parameters (see $\S 3.1$ for details) with both accretion drag and repositioning. In Figure 1 we show the relative separations and velocities of the two SMBHs, and in Figure 2 we show the SMBH growth history. There is no apparent systematic trend based on the particularly choice of implementation.

5 This high gas fraction was chosen to ensure that the primary had a significant gas fraction - in this case $f_{g} \approx 0.3-$ at the time of the SMBH merger. 
TABLE 1

Progenitor Disk Structural Parameters

\begin{tabular}{ccccccc}
\hline \hline & $\begin{array}{c}V_{200} \\
{\left[\mathrm{~km} \mathrm{~s}^{-1}\right]}\end{array}$ & $\begin{array}{c}M_{200} \\
{\left[h^{-1} 10^{10} M_{\odot}\right]}\end{array}$ & $c$ & $\begin{array}{c}h_{D} \\
{\left[h^{-1} \mathrm{kpc}\right]}\end{array}$ & $N_{\text {halo }}$ & $N_{\text {baryons }}$ \\
\hline SB & 160 & 95 & 9 & 4.1 & $3.3 \times 10^{5}$ & $2.0 \times 10^{5}$ \\
SC & 130 & 51 & 10 & 3.2 & $1.8 \times 10^{5}$ & $1.1 \times 10^{5}$ \\
SD & 100 & 23 & 12 & 2.2 & $8.1 \times 10^{4}$ & $4.9 \times 10^{4}$ \\
IM & 80 & 12 & 12 & 1.6 & $4.2 \times 10^{4}$ & $1.5 \times 10^{4}$ \\
\hline \hline
\end{tabular}

Based on this simple experiment, we do not see a systematic trend in the use of accretion drag over repositioning. Rather, the choice of one or the other method introduces a scatter of $\sim 0.1-0.2$ dex, which is noticeably smaller than both the simulation-to-simulation scatter, and even the uncertainty associated with the stochastic nature of SMBH accretion. Therefore, while we note that a more systematic study of SMBH merger prescriptions for hydrodynamical simulations is warranted, our choice of the accretion drag method does not appear to bias our results. However, we do prefer it over repositioning for its numerical stability; in some - admittedly rare - cases, and particularly when the mass ratio of the interaction is high and one galaxy dominates the local potential, the central SMBH in the secondary can decouple from its host galaxy and follows the gravitational field lines of the primary, resulting in a spurious $\mathrm{SMBH}$ merger. However, in general, seeing as the choice of method does not qualitatively change our results, and there is no clear physical argument to favor either, we consider it one of the systematic uncertainties in our modeling.

\subsection{Progenitor Disks}

The progenitor disk models are constructed following Springel et al. (2005). Exponential disks of gas and stars - and optionally a compact bulge - are embedded in a dark matter halo with a Hernquist (1990) density profile, as motivated by cosmological N-body simulations (e.g., Navarro et al. 1996; Bullock et al. 2001; Busha et al. 2005). Their total mass is $V_{200}^{3} /\left(10 G H_{0}\right)$, where $V_{200}$ is the maximum circular velocity at an overdensity of $200 \rho_{c}$, with a disk mass fraction of $m_{d}$, disk gas fraction of $f_{g}=m_{g} /\left(m_{d}+m_{g}\right)$, and a bulge mass fraction of $m_{b}$. The disk scale-length is computed assuming a spin parameter of $\lambda=0.033-0.05$, again motivated by cosmological N-body simulations (e.g., Cole \& Lacey 1996; Vitvitska et al. 2002; Macciò et al. 2007; Bett et al. 2007).

For this work, we use three sets of simulations: (1) major mergers, (2) a mass ratio series, and (3) isolated, bar-unstable disks (hereafter referred to as simply "unstable disks"). The structural parameters of the progenitor disks used in the major merger series are discussed in detail by Robertson et al. (2006c, b) , and we refer the reader to those references for the specifics. The initial disks used in both the mass ratio and unstable disk series are described in Table 11, and are similar to those used in Younger et al. (2007).

\section{SIMULATIONS}

\subsection{Mergers}

The interactions in the major mergers series, and in particular their orbital parameters, are discussed in de-

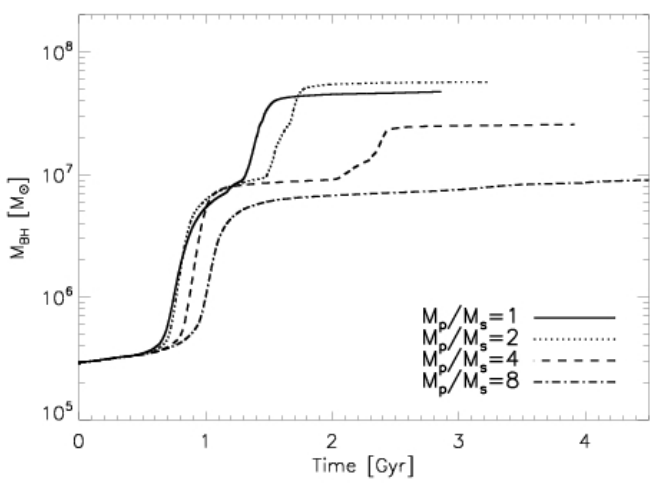

Fig. 3.- SMBH growth histories for four different interactions with mass ratios of: $M_{p} / M_{s} \approx 8$ (dash-double-dot), 4 (dash-dot), 2 (dash), and 1 (major merger; solid). The orbital inclination for each was $i=30^{\circ}$ and the gas fractions of both initial disks $40 \%$ $\left(f_{g}=0.4\right)$. The major mergers clearly grow much more massive SMBHs than do the minor $\left(M_{p} M_{s} \sim 4-8\right)$ mergers.

tail by Robertson et al. (2006b.c), Cox et al. (2006), and Hopkins et al. (2007a); we refer the reader to these papers for a detailed discussion. Each galaxy typically contains $6 \times 10^{5}$ halo particles $\left(0.2 h^{-1} \mathrm{kpc}\right.$ softening $), 2 \times 10^{4}$ bulge particles $\left(0.1 h^{-1} \mathrm{kpc}\right.$ softening), and $4 \times 10^{4}$ stellar disk particles $\left(0.1 h^{-1} \mathrm{kpc}\right.$ softening $)$. The two progenitor galaxies are placed on a zero-energy parabolic orbit, as motived by cosmological N-body simulations (e.g., Benson 2005; Khochfar \& Burkert 2006). We include a range of gas fractions, from relatively gas poor $\left(f_{g}=0.05\right)$ to pure gas disks $\left(f_{g}=1\right)$, covering $\approx 2.5$ dex in total mass - excluded the scaled disks from Robertson et al. (2006c), which are intended to be representative of high redshift systems. We have furthermore varied the resolution by up to a factor of 128 as many particles to confirm that our results are robust.

The mass ratio series (summarized in Table2) includes all permutations of the progenitor disk models in Table 1 . which results in primary to secondary mass ratios ranging from $M_{p} / M_{s} \approx 8$ to major mergers $\left(M_{p} / M_{s}=1\right)$. Both initial disks are dynamically stable over a Hubble time, and have disk mass fractions of $m_{d}=0.05$. The mass resolution of the halo component was $\sim 4 \times 10^{6} M_{\odot}$ per particle $\left(0.15 h^{-1} \mathrm{kpc}\right.$ softening), while the mass resolution of the baryonic component (stars and gas) was $\sim 3.5 \times 10^{5} M_{\odot}$ per particle $\left(0.05 h^{-1} \mathrm{kpc}\right.$ softening $)$ - the particle counts are summarized in Table 2, To simplify the interpretation of the results, none of these models initially had bulges. As before, the two disks are initialized on zero-energy parabolic orbits, with an impact parameter of $\sim h_{D, p}$, where $h_{D, p}$ is the scale length of the primary disk. We also include five different orbital inclinations, as in Younger et al. (2007): $i=0^{\circ}$ (pro- 
TABLE 2

Orbital and Initial Disk Parameters for the Mass Ratio Series

\begin{tabular}{|c|c|c|c|c|}
\hline & Name & $\begin{array}{c}\text { Mass Ratio } \\
M_{p} / M_{s} \\
\end{array}$ & $\begin{array}{c}\text { Inclination } \\
i \\
\end{array}$ & $\begin{array}{c}\text { Gas Fraction } \\
f_{g, p}, f_{g, s} \\
\end{array}$ \\
\hline \multirow[t]{9}{*}{ Standard } & SBfg4Imfg4 & 8 & $0^{\circ}, 30^{\circ}, 90^{\circ}, 150^{\circ}, 180^{\circ}$ & $0.4,0.4$ \\
\hline & SBfg4SDfg4 & 4 & $0^{\circ}, 30^{\circ}, 90^{\circ}, 150^{\circ}, 180^{\circ}$ & $0.4,0.4$ \\
\hline & SBfg 4 Scfg 4 & 2 & $0^{\circ}, 30^{\circ}, 90^{\circ}, 150^{\circ}, 180^{\circ}$ & $0.4,0.4$ \\
\hline & SBfg4SBfg4 & 1 & $0^{\circ}, 30^{\circ}, 90^{\circ}, 150^{\circ}, 180^{\circ}$ & $0.4,0.4$ \\
\hline & Scfg4Imfg4 & 4 & $0^{\circ}, 30^{\circ}, 90^{\circ}, 150^{\circ}, 180^{\circ}$ & $0.4,0.4$ \\
\hline & Scfg4SDfg4 & 2 & $0^{\circ}, 30^{\circ}, 90^{\circ}, 150^{\circ}, 180^{\circ}$ & $0.4,0.4$ \\
\hline & Scfg 4 Scfg 4 & 1 & $0^{\circ}, 30^{\circ}, 90^{\circ}, 150^{\circ}, 180^{\circ}$ & $0.4,0.4$ \\
\hline & SDfg4Imfg4 & 2 & $0^{\circ}, 30^{\circ}, 90^{\circ}, 150^{\circ}, 180^{\circ}$ & $0.4,0.4$ \\
\hline & SDfg4SDfg4 & 1 & $0^{\circ}, 30^{\circ}, 90^{\circ}, 150^{\circ}, 180^{\circ}$ & $0.4,0.4$ \\
\hline Gas-rich & SBfg4Imfg8 & 8 & $30^{\circ}, 150^{\circ}$ & $0.4,0.8$ \\
\hline \multirow{3}{*}{ Secondary } & SBfg4SDfg8 & 4 & $30^{\circ}, 150^{\circ}$ & $0.4,0.8$ \\
\hline & Scfg4Imfg8 & 4 & $30^{\circ}, 150^{\circ}$ & $0.4,0.8$ \\
\hline & Scfg4SDfg8 & 2 & $30^{\circ}, 150^{\circ}$ & $0.4,0.8$ \\
\hline \multirow[t]{9}{*}{ Gas-Rich } & SBfg8Imfg8 & 8 & $30^{\circ}, 150^{\circ}$ & $0.8,0.8$ \\
\hline & SBfg8SDfg8 & 4 & $30^{\circ}, 150^{\circ}$ & $0.8,0.8$ \\
\hline & SBfg8Scfg8 & 2 & $30^{\circ}, 150^{\circ}$ & $0.8,0.8$ \\
\hline & SBfg8Sbfg8 & 1 & $30^{\circ}, 150^{\circ}$ & $0.8,0.8$ \\
\hline & Scfg8Imfg8 & 4 & $30^{\circ}, 150^{\circ}$ & $0.8,0.8$ \\
\hline & Scfg8SDfg8 & 2 & $30^{\circ}, 150^{\circ}$ & $0.8,0.8$ \\
\hline & Scfg8Scfg8 & 1 & $30^{\circ}, 150^{\circ}$ & $0.8,0.8$ \\
\hline & SDfg8Imfg8 & 2 & $30^{\circ}, 150^{\circ}$ & $0.8,0.8$ \\
\hline & SDfg8SDfg8 & 1 & $30^{\circ}, 150^{\circ}$ & $0.8,0.8$ \\
\hline
\end{tabular}

grade coplanar), $30^{\circ}, 90^{\circ}$ (polar), $150^{\circ}$, and $180^{\circ}$ (retrograde coplanar). We consider three different choices for the gas fractions of the initial disks: (1) a standard gas-rich series (as in Robertson et al. 2006c) where both initial disks have $f_{g}=0.4$ over all mass ratios and inclinations, (2) a series with more gas-rich lowermass (SD and IM) secondaries (as suggested by observations; see Roberts \& Haynes 1994; Bell \& de Jong 2000; Schombert et al. 2001; Geha et al. 2006) with $f_{g, p}=0.4$ and $f_{g, s}=0.8$ for a prograde $\left(i=30^{\circ}\right)$ and retrogade $\left(i=150^{\circ}\right)$ interaction, and (3) a high gas fraction series with $f_{g}=0.8$ for both initial disks (which may be representative of higher redshift progenitors; see Erb et al. 2006; Förster Schreiber et al. 2006), over all mass ratios and again for a prograde and retrograde interaction.

The process by which SMBHs grow and self-regulate in major mergers is described in detail by several authors (see e.g., Springel et al. 2005; Hopkins et al. 2005a d, 2006a). Broadly, tidal torques during close passages between disks induce bar instabilities in the stellar distribution. These stellar bars then drain the gas within a critical radius of its angular momentum (Hopkins et al. 2008b) causing it to flow towards the central regions, which in turn fuels a nuclear starburst (see also Barnes \& Hernquist 1991; Mihos \& Hernquist 1994, 1996; Barnes \& Hernquist 1996) and grows the SMBH nearly at an exponential rate. Eventually, when the accretion rate reaches a critical value, feedback terminates the gas inflow and drives a galaxy scale "superwind" that shuts down both star formation and SMBH growth. At the same time, the interaction dynamically heats the stellar component, transforming it into a pressure-supported spheroid: a "red and dead" bulge-dominated galaxy (see also Barnes 1992; Barnes \& Hernquist 1992; Hernquist 1992, 1993).

When the mass ratio is sufficiently high $\left(M_{p} / M_{s} \gtrsim 4\right.$; a "minor merger"), the interaction does not entirely destroy the initial disk (for a detailed discussion for disk survival see Hopkins et al. 2008b), but instead moves a smaller fraction of the stellar mass into a central bulge component (for a more detailed discussion of the kinematics of disk heating by minor mergers, see e.g. Quinn \& Goodman 1986; Quinn et al. 1993; Walker et al. 1996; Velazquez \& White 1999; Kazantzidis et al. 2007). And, in general the nuclear starburst and SMBH accretion do not exhaust the available gas supply, leaving a substantial fraction in the disk component of the remnant (see also Cox et al. 2008, for images and a detailed analysis).

These clear differences motivate a look at the growth history of SMBHs in our simulations, and their sensitivity to the parameters of the interaction. A detailed investigation into the efficiency with which mergers grow black holes, and their luminous lifetimes (see e.g., Hopkins et al. 2005b d, 2006a) for different mass ratios is a topic worthy of more detailed study, which we postpone to future work. However, as a qualitative illustration, we show the growth histories of SMBHs in mergers of different mass ratios and orbital inclinations.

In Figure 3, we see that at fixed inclination and primary galaxy mass, major mergers $\left(M_{p} / M_{s} \sim 2-1\right)$ grow SMBHs that are nearly an order of magnitude more massive than those produced in minor mergers $\left(M_{p} / M_{s} \gtrsim 4\right)$. As shown by Hopkins et al. (2007a) and in subsequent sections of this work, the SMBH traces the binding energy of the bulge. Therefore, the efficiency of SMBH growth in interactions of differing mass ratio is likely a consequence of the efficiency of dynamical heating and bulge formation during those encounters.

Qualitatively, in a minor merger, the first passage induces a bar, gas inflow (Hernquist 1989; Hernquist \& Mihos 1995) and - if it is an interpenetrating encounter - violently relaxing approximately $M_{s}$ of the disk stars, which together form a bulge (see 

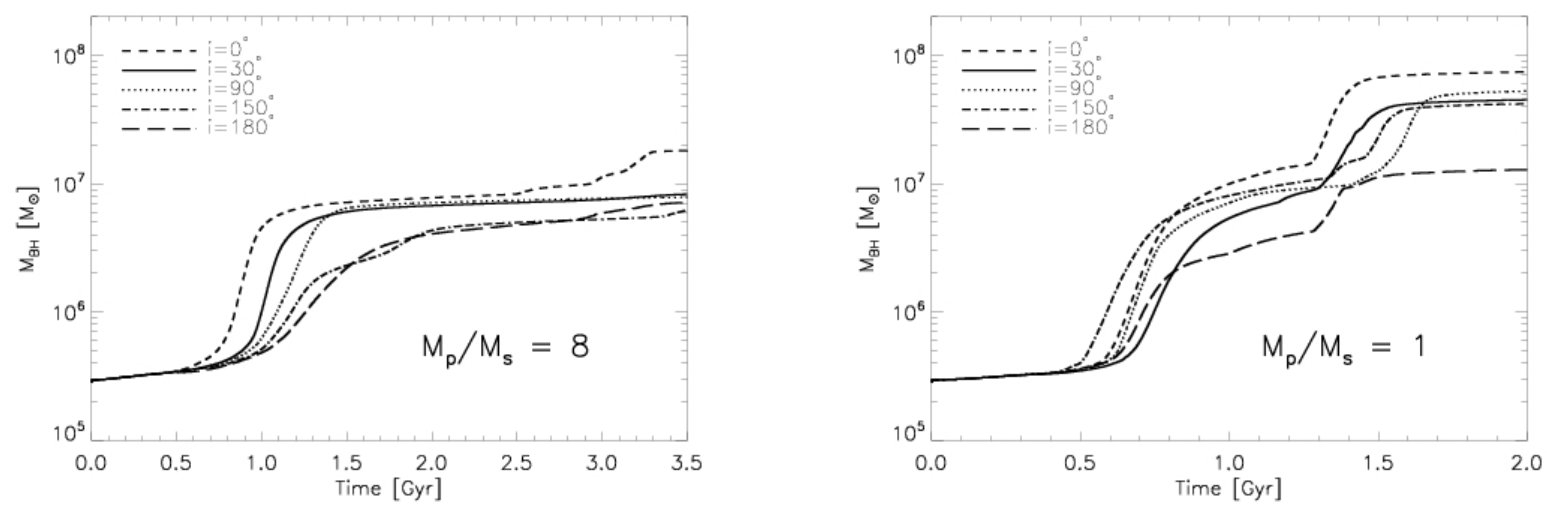

FIG. 4. - SMBH growth histories for two different mass ratio interactions - a major merger $M_{p} / M_{s}=1$ (right panel) and minor merger $M_{p} / M_{s} \approx 8$ (left panel).- for $40 \%$ gas initial disks $\left(f_{g}=0.4\right.$ ) over five different inclinations: $i=0^{\circ}$ (coplanar prograde; dash), 30 $($ solid), $30^{\circ}$ (polar; dot), $150^{\circ}$ (dash-dot), $180^{\circ}$ (coplanar retrograde; dash-double-dot). With the exception of exactly coplanar interactions, which are somewhat pathological, the final SMBH mass is not particularly sensitive to the orbital geometry.

Hopkins et al. 2008b) ${ }^{6}$. It also tends to put the secondary on a mostly radial orbit, and strip a significant fraction of its mass (Bullock \& Johnston 2005; Abadi et al. 2006; Stewart et al. 2007) prior to final coalescence. A low angular momentum final orbit, coupled with a central mass concentration will tend to damp out further bar-driven gas inflows (see e.g., Shen \& Sellwood 2004; Hozumi \& Hernquist 2005; Bournaud et al. 2005; Debattista et al. 2006, and discussion in $\S$ 3.2). This, in combination with less dynamical heating owing to the lower energetics of the interaction, suppresses further bulge growth, and with it the final burst of $\mathrm{SMBH}$ activity seen in major mergers.

In Figure 4, we examine the effect of orbital inclination - prograde coplanar $\left(i=0^{\circ}\right)$ to retrograde coplanar $\left(i=180^{\circ}\right)-$ on both minor (left panel) and major (right panel) mergers. In general, compared to retrograde interactions, prograde encounters produce stronger tidal responses in the disk that induce bars and drive the gas inflows that fuel bulge formation and SMBH growth (see e.g., Cox et al. 2008). We see this effect in our simulations: while the effect is more dramatic for major mergers, for the limiting case of a coplanar interaction, retrograde orbits result in a significantly less massive SMBHs than do prograde. However, when the spins of the two galaxies are not aligned, the resulting $\mathrm{SMBH}$ is largely independent of orbital inclination.

These trends with mass fraction and orbital inclination qualitatively motivate the aim of this work: given a particular encounter, what mechanism sets the final SMBH mass?

\subsection{Disk Instabilities}

\footnotetext{
6 This suggests that as the impact parameter increases, the resultant bulge will tend more towards a pseudobulge. In fact, since no galaxy is truly isolated, the unstable disks outlined in $\S 3.2$ can be thought of as the limiting case of low mass satellites and halo substructure seeding the bar instabilities via flyby encounters. We have run a limited experiment, varying the impact parameter for a small subset of interactions and find evidence that the structural properties of the resultant bulges do tend towards pseudobulges for very large impact parameters (including the normalization offset in $M_{B H}-\sigma$; see $\S 4.3$ and 5.3 . However, a systematic investigation into the dependence of the structural properties of the bulge on the orbital parameters of the interaction is outside the scope of this paper, and thus we defer it to future work.
}

The simulations in the unstable disk series evolve variants of the same disk models in isolation, the parameters of which are summarized in Table 3. The mass resolution and softening lengths of the particles were kept the same as with the mass ratio series, and the particle counts are summarized in Table 4. The disk mass fraction is increased while holding the total mass fixed, which seeds global instabilities by increasing the selfgravity of the disk relative to its kinetic energy from rotation (see Ostriker \& Peebles 1973; Efstathiou et al. 1982). For three initial disk models (SB, Sc, and SD), we consider two disk mass fractions $\left(m_{d}=0.08\right.$ and 0.10$)$, and three gas fractions $\left(f_{g}=0.4,0.6\right.$, and 0.8$)$. For a subset of these simulations, we consider three different seed black hole masses $-M_{B H, i}=10^{4}, 10^{5}$, and $10^{6} h^{-1}$ $M_{\odot}-$ and two different equations of state $-q_{E O S}=0.5$, 1.0 .

Stellar bars are found generically in numerical simulations of isolated disk galaxies (Hohl 1971), and represent a global instability in which rotating disks swingamplify spiral density wave perturbations (Toomre 1981; Binnev \& Tremaine 1987). They have been shown empirically to develop when the self-gravity of the disk is comparable to its kinetic energy of rotation (Ostriker \& Peebles 1973; Efstathiou et al. 1982). Most disk galaxies in the local universe have bars (e.g., Eskridge et al. 2000; Menéndez-Delmestre et al. 2007), and - despite conflicting observational results - it seems clear that a substantial fraction of high redshift $z \sim 1$ disks also show bar features (e.g., Jogee et al. 2004; Sheth et al. 2008). These bars have been shown both theoretically (Athanassoula 1992, 2000) and observationally to drive gas inflows and nuclear starbursts (Jogee et al. 1999, 2002, 2005; Petitpas \& Wilson 2002, 2003). As a result, they represent a potentially promising mechanism for fueling the growth of nuclear SMBHs, which has at times been invoked in semi-analytic models (e.g., Bower et al. 2006) as their dominant mode of growth.

Since the onset of the instability is a collisionless process (see Toomre 1981; Binney \& Tremaine 1987), it develops first in the stellar distribution. The stellar bar then drains the gas within a critical radius of its angular momentum (Barnes \& Hernquist 1996; Hopkins et al. 2008b), driving it inwards fueling a nuclear starburst; a 
TABLE 3

Initial Parameters for the Unstable Disk Series

\begin{tabular}{cccccc}
\hline \hline & Name & $\begin{array}{c}\text { Disk Mass Fraction } \\
m_{d}\end{array}$ & $\begin{array}{c}\text { Initial Gas Fraction } \\
f_{g}\end{array}$ & $\begin{array}{c}\text { Seed BH Mass } \\
M_{B H, i} /\left(h^{-1} M_{\odot}\right)\end{array}$ & $q_{E O S}$ \\
\hline \multirow{2}{*}{ Standard } & USB08 & 0.08 & $0.4,0.6,0.8$ & $10^{5}$ & 1.0 \\
& USc08 & 0.08 & $0.4,0.6,0.8$ & $10^{5}$ & 1.0 \\
& USD08 & 0.08 & $0.4,0.6,0.8$ & $10^{5}$ & 1.0 \\
& USB10 & 0.10 & $0.4,0.6,0.8$ & $10^{5}$ & 1.0 \\
& USc10 & 0.10 & $0.4,0.6,0.8$ & $10^{5}$ & 1.0 \\
& USD10 & 0.10 & $0.4,0.6,0.8$ & $10^{5}$ & 1.0 \\
\hline BH Seed Mass & USSB10 & 0.10 & 0.4 & $10^{6}$ & 1.0 \\
& USSC10 & 0.10 & 0.4 & $10^{6}$ & 1.0 \\
& USSD10 & 0.10 & 0.4 & $10^{4}$ & 1.0 \\
\hline \multirow{2}{*}{ EOS } & USSB10 & 0.10 & 0.4 & $10^{5}$ & 0.5 \\
& USSc10 & 0.10 & 0.4 & $10^{5}$ & 0.5 \\
& USSD10 & 0.10 & 0.4 & $10^{5}$ & 0.5 \\
\hline
\end{tabular}

TABLE 4

Resolution of the Unstable Disk Series

\begin{tabular}{ccc}
\hline \hline Name & $N_{\text {halo }}$ & $N_{\text {baryons }}$ \\
\hline USB08 & $3.2 \times 10^{5}$ & $3.2 \times 10^{5}$ \\
USC08 & $1.7 \times 10^{5}$ & $1.7 \times 10^{5}$ \\
USD08 & $7.9 \times 10^{4}$ & $7.8 \times 10^{4}$ \\
USB10 & $3.2 \times 10^{5}$ & $4.0 \times 10^{5}$ \\
USc10 & $1.7 \times 10^{5}$ & $2.1 \times 10^{5}$ \\
USD10 & $7.7 \times 10^{4}$ & $9.8 \times 10^{4}$ \\
\hline
\end{tabular}

process analogous to merger-driven gas inflows in which the bar is induced by the time-evolution of the tidal field during close passages (see Mihos \& Hernquist 1994). This nuclear starburst concentrates some of the mass of the initial disk at its center, forming a pseudobulge (see Kormendy \& Kennicutt 2004a, for a review) and eventually leading to the destruction of the bar over a relatively short timescale as the central mass concentration damps out the spiral density waves $(\sim 1-2$ Gyr; Shen \& Sellwood 2004; Hozumi \& Hernquist 2005; Bournaud et al. 2005; Debattista et al. 2006). Because there is no interpenetration by a satellite, there is correspondingly no violent relaxation of the stellar disk and as a result the pseudobulge is almost entirely dominated by stars formed during the simulation.

Though the feedback model employed for the unstable disk series is identical in both implementation and parameter choice to that used for the major mergers and mass ratio series, in order to insure a fair comparison it is important that the growth of the SMBH be selfregulated by feedback rather than limited by gas depletion in the central regions. This will be the case if the stellar bars, like those induced during close passages during major and minor mergers, drive gas inflows that create a central gas reservoir many times more massive than the SMBH and a substantial density enhancement in its immediate vicinity, which will result in Eddingtonlimited growth until the local gas supply is then expelled by a thermal feedback driven blast wave. In Figure 5 we show the gas inflow - and feedback driven outflow as a function of time for a representative unstable disk simulation. We find that the gas inflows driven by the stellar bar concentrates a significant gas supply in the central several hundreds of parsecs. Furthermore, in Fig-

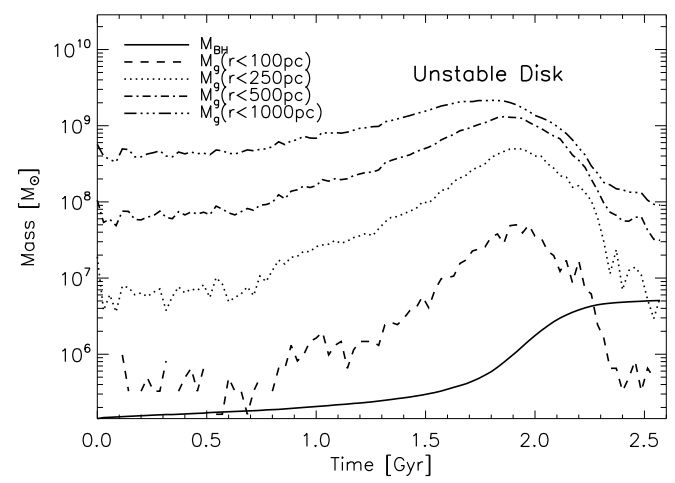

FIG. 5.- Illustration of the bar-driven gas inflows and resulting feedback driven outflows in a representative unstable disk simulation: USB08 with baryon fraction $m_{d}=0.08$ and initial gas fraction $f_{g}=0.4$. Shown is the SMBH mass (solid line) and total gas mass $\left(M_{g}\right)$ within 100 (dashed line), 250 (dotted line), 500 (dash-dotted line), and 1000 (dash-triple-dotted line) parsecs. The stellar bar efficiently concentrates a significant gas reservoir - several hundreds $\times M_{B H}$ - within a few hundred parsecs of the SMBH which are subsequently expelled via feedback driven outflows.

ure 6] we confirm that the gas density enhancement in the immediate vicinity of the SMBH - which is used to calculate the instantaneous accretion rate - is comparable to those produced in merger-driven inflows. Finally, we find that the accretion rate - i.e., AGN light curve - decays rapidly with a power-law slope similar to that expected from feedback driven outflows (e.g., Hopkins \& Hernquist 2006). As a result, we confirm that SMBH growth in our unstable disk simulations is regulated by feedback rather than gas depletion, and thus - if we assume that accretion and feedback in mergers and unstable disks are similar - our feedback model is 


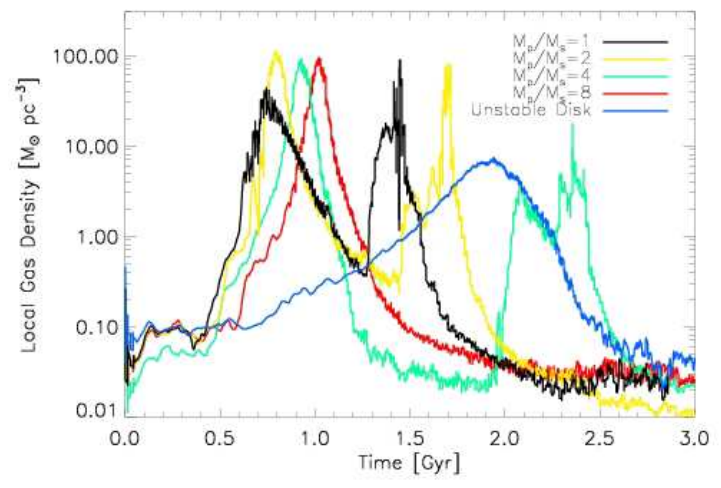

FIG. 6. - Local gas density - the mean density over one SPH smoothing length of 64 particles - near one of the SMBHs in four merger simulations of varying mass ratio $-M_{p} / M_{s}=1$ (black line), 2 (yellow line), 4 (green line), and 8 (red line), all with an orbital inclination of $i=30^{\circ}$ and initial gas fraction $f_{g}=0.4-$ as compared to a representative unstable disk simulation (same as in Figure 5 blue line). The local gas densities, which are used to calculate the instantaneous accretion rate of the $\mathrm{SMBH}$, are comparable in mergers and unstable disks.

appropriate to these systems.

\subsection{Systematic Uncertainties}

Robertson et al. (2006c) and Hopkins et al. (2007a) both comment on the sensitivity of the predicted SMBH scalings arising from major mergers to choices in our models: in particular the choice of the initial seed mass $M_{B H, i}$ and the EOS parameter $q_{E O S}$, which unlike $f_{g}$ do not have a clear observationally preferred, physically motivated value. These authors find that over a relatively wide range of values for these parameters, the scaling relations with final SMBH are well within the scatter both in the observed and simulated relations. Therefore, while the overall growth history of an individual simulation may change significantly with specific choices for these parameters, the predicted scalings remain statistically unchanged. This is consistent with our interpretation of the scaling of SMBH mass with the binding energy of the bulge as the more fundamental, physical relation; the parameters of the bulge component of the remnant are relatively insensitive to these parameters.

Because the mass ratio series is consistent with the scalings derived from major mergers, we are confident that they too are not affected by these systematic uncertainties in our modeling. However, it is not clear $a$ priori how varying $M_{B H, i}$ and $q_{E O S}$ will affect SMBHs grown in unstable disks. This is because the SMBH does not grow by more than one or two orders of magnitude and the stochastic nature of its accretion may make it more sensitive to the details of the growth history than SMBHs grown via major or even minor mergers, which accrete several orders of magnitude more at comparable total baryonic mass (see, e.g. Figure 3).

In Figure 7 we show the growth history of SMBHs in three different disk models with $f_{g}=0.4$ for different seed masses: USB10 $\left(M_{B H, i}=10^{5}, 10^{6} M_{\odot} / h\right)$, $\mathrm{USC10}\left(M_{B H, i}=10^{5}, 10^{6} M_{\odot} / h\right)$, and USD10 $\left(M_{B H, i}=\right.$ $\left.10^{4}, 10^{5} M_{\odot} / h\right)$. We find that, as with the gas supply, while the detailed evolution differs substantially, the final SMBH masses are largely consistent to within the scatter in the simulated and observed relations. The largest dif-

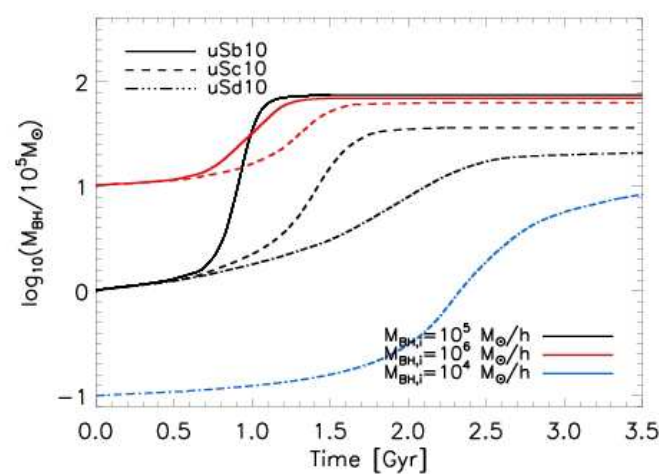

FIG. 7.- SMBH growth histories for three different disk models - SB (solid line), SC (dashed line), and SD (dot-dot-dashed line) and three different SMBH seed masses $-M_{B H, i}=10^{4}$ (blue), $10^{5}$ (black), and $10^{6}$ (red) $h^{-1} M_{\odot}$. We find that, despite differences in the SMBH growth histories, there is no systematic trend between the final SMBH mass and $M_{B H, i}$, and that the maximum deviation for an order of magnitude difference in the seed mass is only $\sim 0.3$ dex.

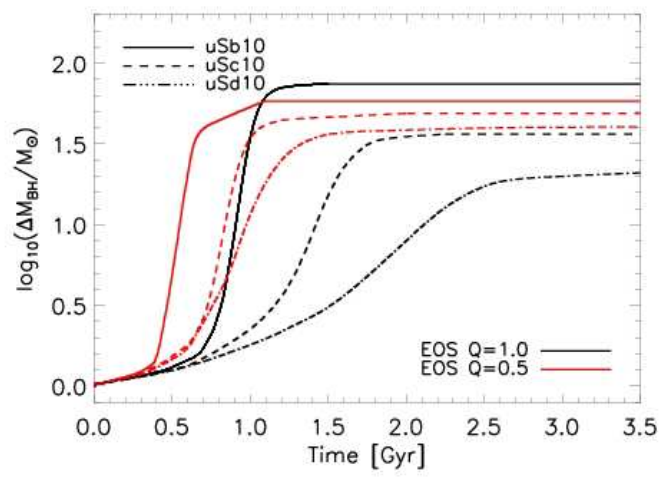

FIG. 8. - SMBH growth histories for three different disk models - SB (solid line), SC (dashed line), and SD (dot-dot-dashed line) - and three different SMBH seed masses $-q_{E O S}=0.5$ (red) and 1.0 (black). We find - as with the initial seed SMBH mass - despite differences in the growth histories there is no systematic trend between the final SMBH mass and the stiffness of the EOS.

ference $(\sim 0.3 \mathrm{dex})$ is for the lowest mass disk model, for which the SMBH accretes the least mass and is therefore more sensitive to certain choices of parameters for our modeling.

In Figure 8, we show the SMBH growth history for different effective equation of state parameters $q_{E O S}=0.5$ and 1.0 for three different disk models with $f_{g}=0.4$. As $q_{E O S}$ approaches unity, it increases the dynamical stability of the gas against Toomre (1964) instabilities (see Robertson et al. 2004, 2006c). This effectively delays the onset of the bar-driven inflow in disk models with $q_{E O S}=1.0$ relative to those with a softer EOS $q_{E O S}=0.5$. However, despite these differences in the growth history of the SMBHs, their final masses are roughly consistent with one another to within the scatter in the observed and simulated relations. The largest differences, as with different seed masses, are for the lowest mass disk model ( $\sim 0.3 \mathrm{dex})$. More important, there are no systematic trends with $q_{E O S}$ : the final SMBH mass in uSb10 is somewhat lower, while those in uSc10 and Sd10 are slightly higher. 


\subsection{Remnant Properties}

The structural and kinematic properties of the remnant were extracted after the system reached a state of approximate dynamical equilibrium; for the major and mass ratio series, this was typically $\sim 1 h^{-1}$ Gyr after the final coalescence of the two SMBHs, while for the unstable disks this was typically $\sim 0.5 h^{-1}$ Gyr after the peak of the SMBH accretion rate. If the remnant was diskdominated, we then fit a combination exponential and Sersic (1968) profile to the projected stellar mass density (i.e., "surface brightness") profile along 64 lines of sight $^{7}$, from which we estimated the median bulge mass $\left(M_{b}\right)$ and effective radius $\left(R_{e}\right)$. If the remnant was bulgedominated ( $\mathrm{B} / \mathrm{T} \gtrsim 0.8)$, we took $M_{b}$ to be the total stellar mass, and $R_{e}$ the half-mass radius. The inner velocity dispersion $(\sigma)$ was measured within $R_{e}$, again taking the median along 64 different lines of sight. This procedure was identical between the mergers and unstable disks. Furthermore, Hopkins et al. (2008b) show that surface brightness fitting of the kind employed in this work recovers on average the bulge-to-disk ratios inferred from a kinematic decomposition of the stellar particles.

\section{RESULTS}

\subsection{The Black Hole Fundamental Plane}

Hopkins et al. (2007a) find that there exist correlations in the residuals of fits such as the $M_{B H}-\sigma$ and Magorrian et al. (1998) relations; for example, at fixed $\sigma$ the residual SMBH mass scales approximately as $\sim M_{b}^{0.72}$, and at fixed $M_{b}$ the residuals scale as $\sim \sigma^{1.40}$. By marginalizing over two parameters $-M_{b}$ and either $\sigma$ or $R_{e}$ - they found a best-fit BHFP (analogous to that observed for elliptical galaxies; Dressler et al. 1987; Djorgovski \& Davis 1987) which minimized these residual correlations with the form $M_{B H} \sim M_{b}^{0.72} \sigma^{1.4}$, both in simulations (Hopkins et al. 2007a) and for observed systems (Barway \& Kembhavi 2007; Hopkins et al. 2007b; Aller \& Richstone 2007). This is statistically indistinguishable from a correlation with the bulge binding energy proxy $M_{b} \sigma^{2}\left(M_{B H} \sim\left(M_{b} \sigma^{2}\right)^{0.7}\right)$, and can be interpreted as reflecting the nature of feedback regulated SMBH growth: accretion accelerates until feedback is sufficient to unbind the local gas supply, abruptly terminating the inflow and cutting off further growth. Therefore it is more "fundamental" than its various projections, such as the $M_{B H}-\sigma$ and Magorrian et al. (1998) relations - a point we discuss in more detail in $\S 5.1$.

To systematically test this hypothesis, we examine the binding energy correlation - which is statistically equivalent to the BHFP - in three different modes of SMBH fueling: major mergers, minor mergers, and unstable disks (see $\S 3$ for details). Figure 9 shows the binding energy correlation for major mergers (grey hexagons), the mass ratio series (left: colored points; right: black triangles), and unstable disks (right: colored points), along with the

\footnotetext{
7 The detailed formation and growth of stellar bulges via minor mergers is certainly an interesting problem in its own right, and the simulations presented here are ideal for just such an analysis. However, for this work we restrict ourselves to simple profile fits and bulge masses for both the purposes of clarity and to best approximate observational estimates of bulge masses, and postpone a detailed analysis of the bulge structure and kinematics to a future paper (Cox et al. 2008, in preparation)
}

observations listed in Hopkins et al. (2007b). We find that over a range of baryonic masses, gas fractions, and orbital parameters they all lie along the same relation to within the scatter, and that all reproduce the observed correlation.

\subsection{The Role of the Fuel Supply}

Inasmuch as their growth is terminated at a critical accretion rate, the final masses of SMBHs should not be determined by the available fuel supply so long as the gas reservoir is much more massive than the SMBH. While it is the case that the final SMBH mass is strongly correlated with the total gas mass of the system, this reflects the structural properties of bulges in gas-rich merger remnants: owing the effects of dissipation, a more gas rich progenitor will lead to a more compact bulge at fixed total mass, and consequently a deeper central potential $\phi_{c}$ and velocity dispersion $\sigma$ (see e.g., Robertson et al. 2006b. c; Hopkins et al. 2007a). As shown by Hopkins et al. (2007a) for major mergers, at fixed potential the gas fraction has no effect on SMBH growth.

One method of illustrating this is presented in Figure 10, which shows the BHFP and binding energy correlations for the mass ratio series (colored points) as compared to those derived from simulations of major mergers (grey hexagons; solid line with the dotted line indicating the scatter). Here we show that increasing the initial gas fraction from $f_{g}=0.4$ to 0.8 for the identical interaction will drive the remnant along the BHFP and binding energy correlations, but not systematically away from them. Therefore, we find that the typically more massive SMBHs resulting from simulations of more gas-rich encounters is related to the structural properties of the resulting remnant - which is more compact at fixed $M_{b}$ (Hopkins et al. 2008c, $\mathrm{f}, \mathrm{e}, \mathrm{g}, \mathrm{h}$ ), and thus has larger binding energy - than it is to the larger available fuel supply, leaving the nature of these correlations unchanged.

In Figure 11, we show the residual correlation of $\mathrm{SMBH}$ mass at fixed binding energy with the gas fraction at the peak of the starburst $\left(f_{\text {g,peak }}\right)$ for both mergers (left) and unstable disks (right). We find no correlation between

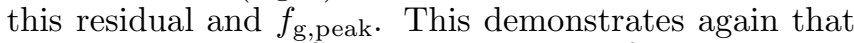
to the extent that SMBH growth is self-regulated, its final mass is not correlated with the available fuel supply but rather with the binding energy of its host bulge.

\subsection{The Projected Correlations}

While the BHFP and binding energy scalings with SMBH mass are consistent between mergers and unstable disks, individual projections of these relations are not consistent. In Figures [12] and [13] we show the predicted $M_{B H}-\sigma$ and Magorrian et al. (1998) scalings from the unstable disk simulations, as compared to both the major merger and mass ratio series. One of the more striking features of these results is the normalization offset in the $M_{B H}-\sigma$ relation, in which SMBHs grown via disk instabilities are less massive at fixed $\sigma$ than those grown in mergers but maintain a similar slope. Or conversely, the bulges grown via disk instabilities are more compact at fixed SMBH and bulge mass.

This difference was noted by $\mathrm{Hu}$ (2008) and Graham (2008) as a normalization offset between the $M_{B H}-\sigma$ 

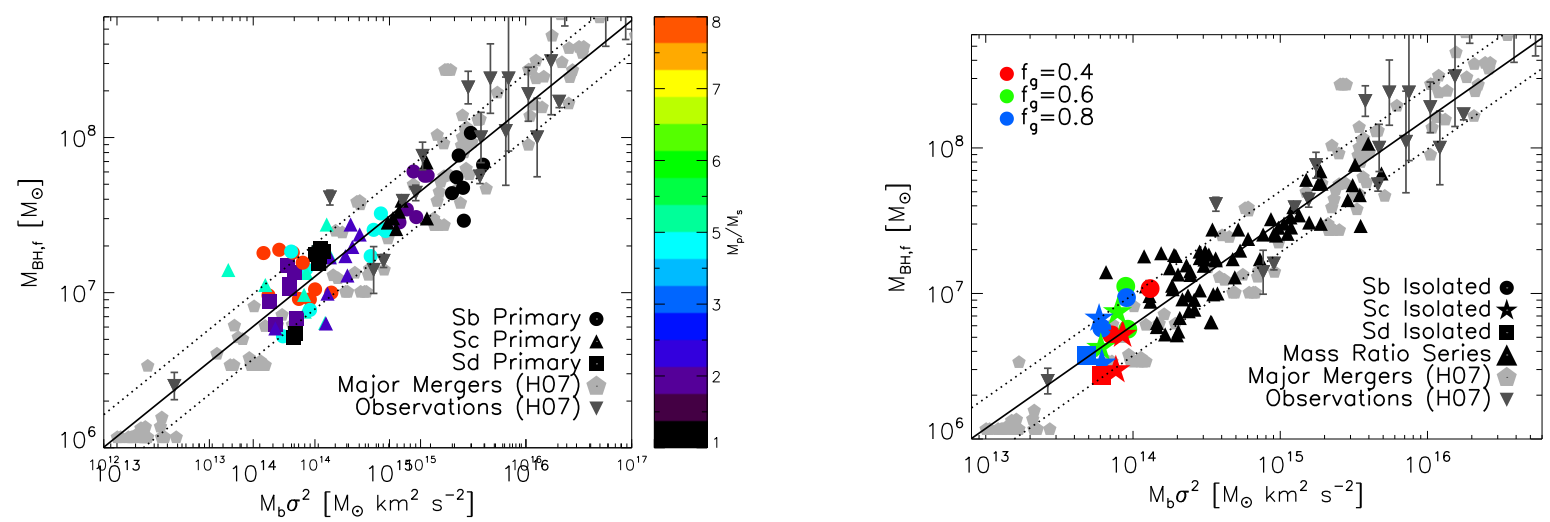

FIG. 9. - Left: Binding energy correlation - which is statistically equivalent to the BHFP (H07; Hopkins et al. 2007a) - for the major and mass ratio series (solid line, scatter indicated by dotted lines). The grey hexagons are the major mergers series and the grey inverted triangles are observations, both from H07. The mass ratio series - including all gas fractions - are colored according to the mass ratio (see color bar to the right of the figure) with the primary galaxy model indicated according to: SB (filled circle), SC (filled triangles), and SD (filled square). Right: The binding energy correlation for mergers (see previous; the mass ratio series as filled triangles) and unstable disks (same labeling as primary galaxy in previous) for three different gas fractions: $f_{g}=0.4$ (red), 0.6 (green), and 0.8 (blue). We find that SMBHs grown via major mergers, minor mergers, and disk instabilities all lie along the same BHFP and binding energy correlations.

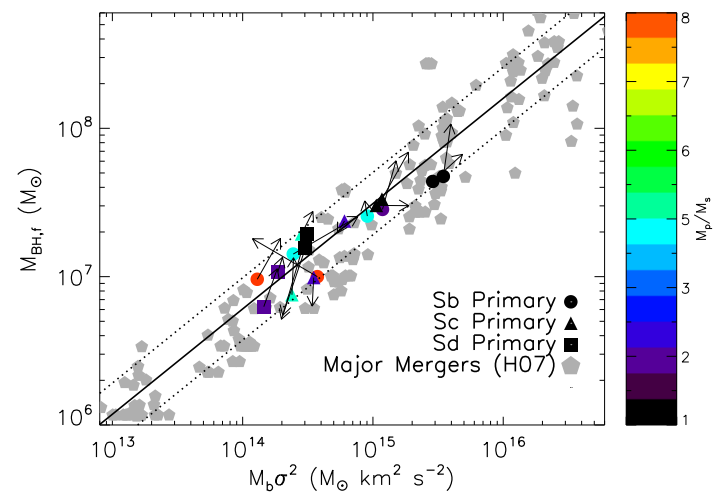

FIG. 10.- Influence of gas fraction on the location of SMBHs on the binding energy correlation for the mass ratio series. The labeling is the same as the left panel of Figure 9 Shown are the mass ratio series simulations with initial gas fraction $f_{g}=0.4$, and the arrows indicate the location of identical interactions with more gas rich progenitors $\left(f_{g}=0.8\right)$. We find that increasing the gas content of the progenitors drives these systems along these relations - owing to the structural properties of bulges formed during more gas-rich interactions (see e.g., Robertson et al. 2006a b) - but not systematically away from them. This reflects the different structural properties of bulges formed from more gas-rich disks, leaving the fundamental character of the BHFP and binding energy correlations unchanged.

relations in "classical" bulges, which are likely the result of mergers, and "pseudobulges" or bulges in barred systems which were likely grown via secular processes (Kormendy \& Kennicutt 2004b). Our simulations agree quite well with these observational results. $\mathrm{Hu}(2008)$ interprets this result as possible evidence for less efficient fueling of SMBHs via bar instabilities, creating a less massive SMBH at fixed $\sigma$. However, we find that this reflects structural differences between bulges produced in merger simulations versus those produced via secular processes in the unstable disks: bulges and pseudobulges $^{8}$ lie along different Faber \& Jackson (1976) re-

8 The exact location of the pseudobulges produced in unstable disks on the Faber \& Jackson (1976) relation is somewhat sensitive to the initial conditions. However, their manifestly different structural properties relative to classical bulges produced via mergers is a robust result. lations (see Figure 14). These systems all lie along the same BHFP and binding energy correlations, suggesting that the lower mass SMBHs found in pseudobulges at fixed $\sigma$ reflect their lower overall binding energy owing to structural differences rather than the efficiency of fueling. These structural differences are driven largely by the different formation mechanism of pseudobulges, which retain a great deal more rotation than their classical analogues - the LOS velocity dispersion will include rotational motions. In addition, because all the mergers presented in this work coalesce, the structural differences can further be understood via simple energy arguments: in a merger, conservation of kinetic energy requires that the effective radius of the bulge increase - and therefore the velocity dispersion must decrease - when the system has fully merged (e.g., Hernquist et al. 1993).

\section{DISCUSSION}

\subsection{The "Fundamental" Character of the BHFP}

Among local systems, there are a relatively large number of tight correlations between SMBHs and the properties of their host galaxies (for reviews, see e.g., Novak et al. 2006; Hopkins et al. 2007a; Graham 2008). The question of which of these is more "fundamental" has two components: (1) which is a better predictor of SMBH mass in systems for which detailed measurements are not feasible, and (2) which better reflects the physical mechanism driving the co-evolution of SMBHs and bulges. The answer to (1) is largely determined by observational uncertainty and sample selection, and is outside the scope and limits of this investigation. However, (2) is directly addressed by our simulations, and highlights the physical mechanisms causing the self-regulated growth of SMBHs.

Graham (2008) argues that the residual-residual correlations that initially motivated the observed and simulated BHFPs are a consequence of sample selection, which includes bulges produced via both interactions and secular processes. In particular, the author notes that when barred systems are excluded, these correlations are no longer significant. This is entirely consistent with expectations from our modeling. Owing to the relatively 

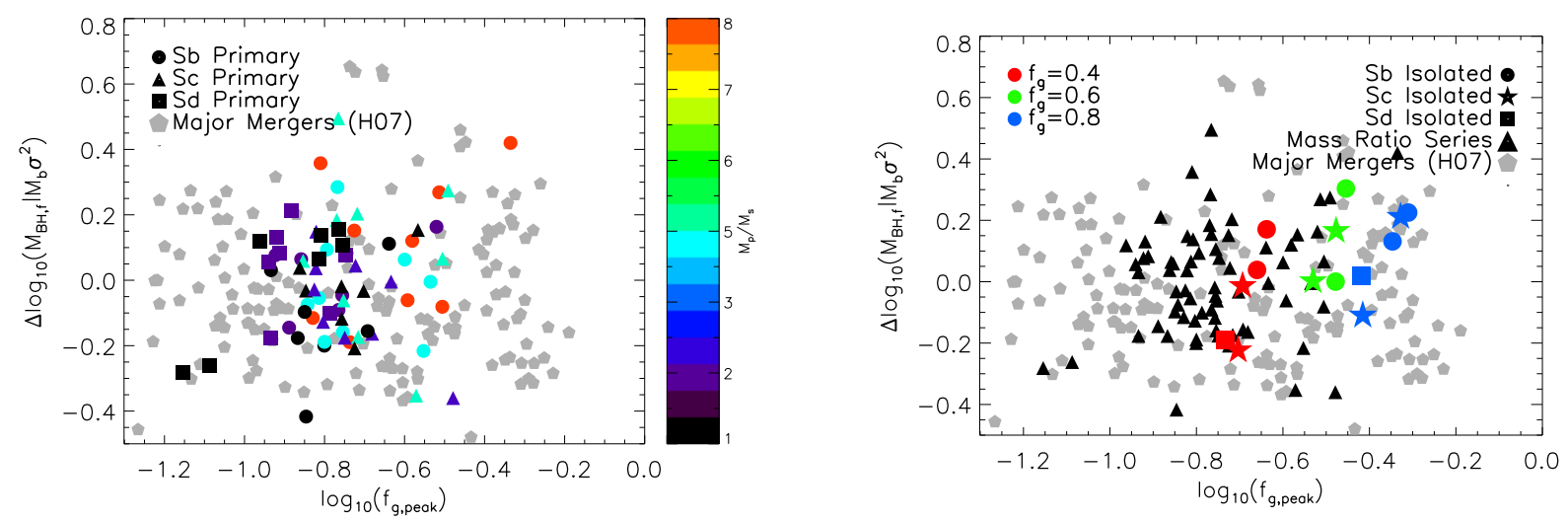

FIG. 11. - Correlation between the SMBH mass residual at fixed binding energy and the gas fraction at the peak of the starburst $\left(f_{\mathrm{g}, \mathrm{peak}}\right)$ for mergers (left) and unstable disks (right), with the same labeling as Figure 9 We find that these residuals are uncorrelated in all three sets of simulations, indicating that the final SMBH mass is not determined by the available fuel supply.
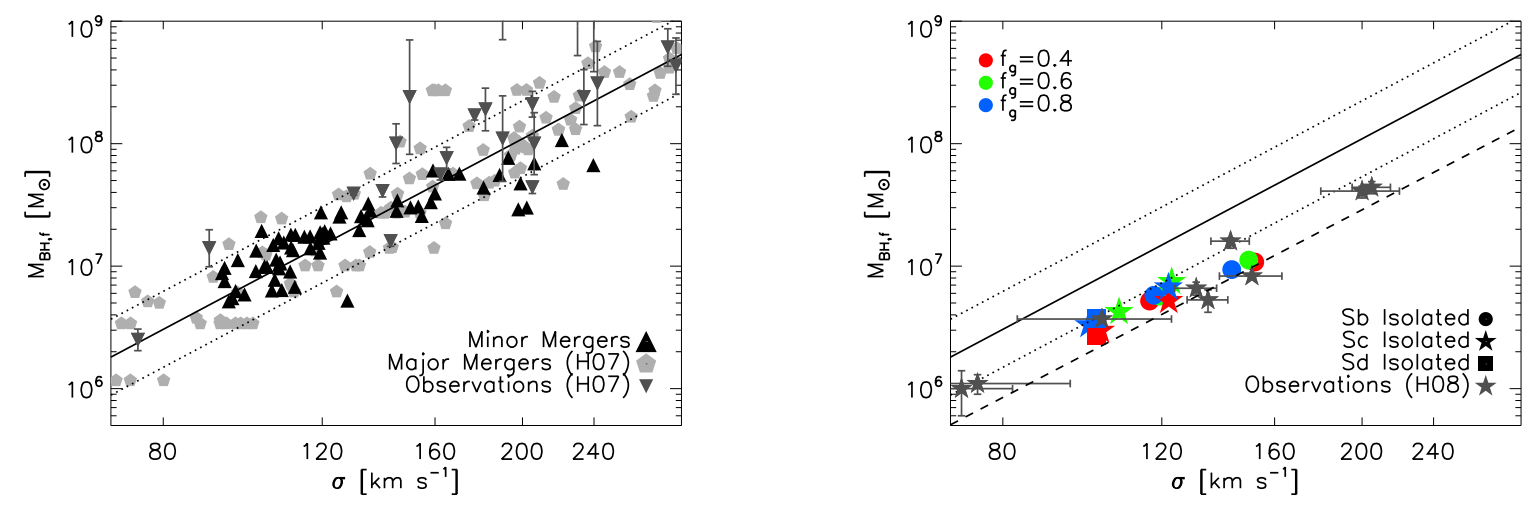

Fig. 12.- $M_{B H}-\sigma$ relation for mergers (left) and unstable disks (right), with the same labeling as the right hand panel of Figure 9 The black solid and dashed lines indicate the best-fit relations and scatter from H07, and we include observations of SMBHs hosted by pseudobulges - which are likely grown via disk instabilities (see Kormendv \& Kennicutt 2004a) - from Hu (H08; grey stars indicate data, dashed line indicates best-fit relation; 2008).

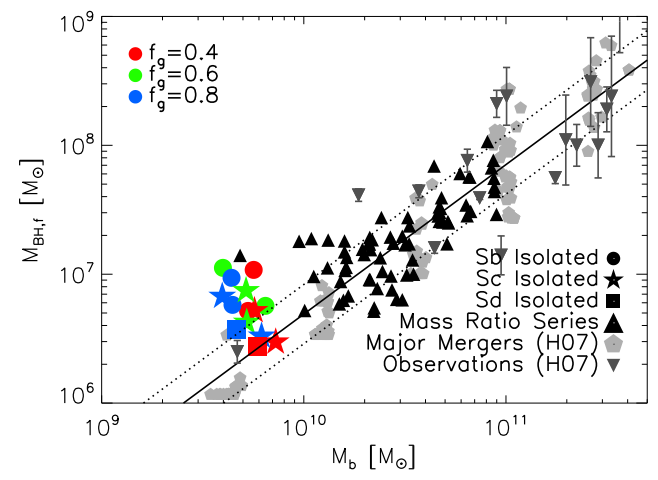

FIG. 13. - Magorrian et al. (1998) relation for mergers and unstable disks, with the same labeling as Figure 12

small dynamic range probed by classical bulges with robust SMBH mass measurements, we would not necessarily expect the residual correlations to appear significant without the inclusion of lower mass systems, which are more likely to be barred. More important, the correlation between residuals will be most apparent in systems with different structural properties, like classical bulges and massive ellipticals as compared to pseudobulges and barred systems.

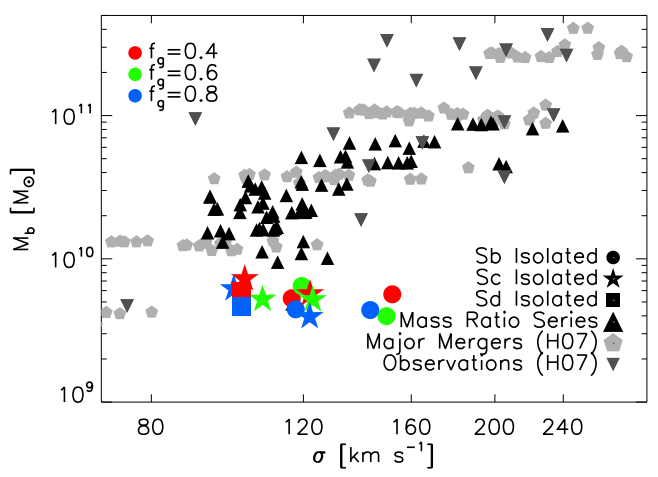

Fig. 14. - Faber \& Jackson (1976) relation for bulges grown via mergers and disk instabilities, with the same labeling as the right hand panel of Figure 9 Bulges produced via major and minor mergers ("classical bulges") lie along the same correlation, while those produced via secular processes ("pseudobulges"; Kormendy \& Kennicutt 2004a) follow a different relation. This demonstrates that these two types of bulges are structurally different; plausible, given their likely different formation mechanisms.

Moreover, our results suggest that while the results of Graham (2008) directly address (1), they do not provide convincing evidence against the BHFP and binding energy argument as the solution to (2). In Figure 9, we 

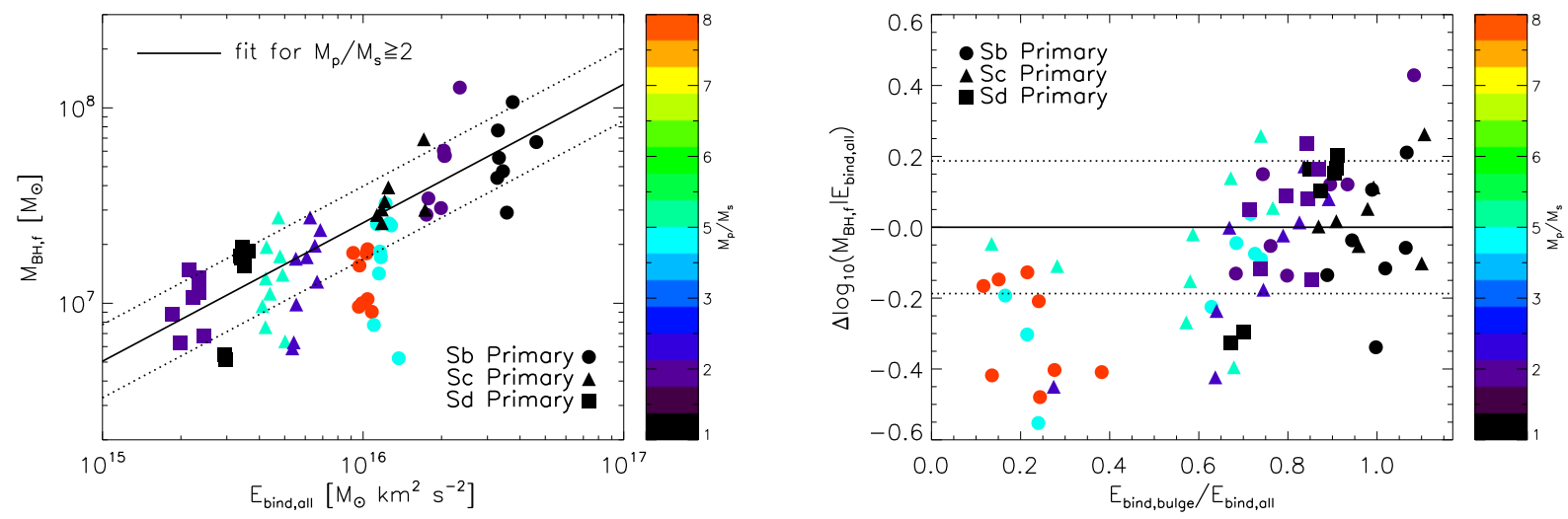

FIG. 15.- Left: Correlation between SMBH mass and the binding energy of all the stellar particles $E_{\text {a,bind }}$, for the mass ratio series. Labeling is the same as the left hand panel of Figure 9 The solid line represents a fit to the relation for major mergers $\left(M_{p} / M_{s} \lesssim 2\right)$, which are bulge-dominated, with the r.m.s. scatter indicated by dotted lines. Right: The residual SMBH mass at fixed $E_{\text {all,bind }}$, as compared to $E_{\mathrm{b}, \text { bind }} / E_{\text {all,bind }}$ using the relation derived for major mergers (see left), where $E_{\mathrm{bulge}}$,bind $\approx 10.1 M_{b} \sigma^{2}$ is the approximate total binding energy assuming a spherical Hernquist (1990) profile. We find a significant residual correlation as $E_{\mathrm{b}, \text { bind }} / E_{\text {all,bind }}$ approaches unity, suggesting that the bulge binding energy is a better predictor of final SMBH mass than the total stellar binding energy in systems that retain a significant disk component (minor mergers; $M_{p} / M_{s} \gtrsim 4$ ); i.e., the SMBH "sees" the bulge more so than the overall stellar distribution.

show that simulations of three different fueling mechanisms - major mergers, minor mergers, and disk instabilities - all reproduce the observed BHFP and binding energy correlations. This suggests that in all three cases, the mass of the SMBH is set by the critical accretion rate at which feedback terminates the gas inflow, and halts further growth of the SMBH. At the same time, SMBHs grown in unstable disks versus interactions lie along different projected correlations in Figures 12 and [13. In this way, the BHFP and binding energy correlations are more fundamental in that they reflect the feedback regulated growth of SMBHs across bulges with very different structural properties.

\subsection{Implications for SMBH Fueling Models}

Our modeling demonstrates that in feedback-regulated models of SMBH growth, while the BHFP and binding energy correlations are universal, their projections - $M_{B H}-\sigma$, Magorrian et al. (1998), etc. - are sensitive to the structural properties of the spheroidal component of the host galaxy. For the particular case of $M_{B H}-\sigma$, this results in a normalization (see $\S 4.3$ and Figure 12) offset between classical bulges - which were likely formed during mergers - and pseudobulges and those found in barred systems - which are likely produced via secular processes. Given that our particular implementation offers a good approximation to the effects of feedback more broadly (see $\S 2.2$ ), this is a generic prediction of models in which SMBH growth is self-regulated via feedback.

Consequently, that both the BHFP (Aller \& Richstone 2007; Hopkins et al. 2007b) and an apparent offset in $M_{B H}-\sigma$ between classical and secular bulges $(\mathrm{Hu}$ 2008; Graham 2008) are observed provides evidence for feedback regulated models more generally (Silk \& Rees 1998; King 2003, 2005; Murrav et al. 2005; Sazonov et al. 2005; Robertson et al. 2006c; Thacker et al. 2006; Hopkins et al. 2007a). It also presents a challenge to alternative models in which the projected scalings are relatively insensitive to the structural properties of the spheroid, including: stellar capture by the accretion disk (Zhao et al. 2002; Miralda-Escudé \& Kollmeier
2005), regulation via a viscous star-forming accretion disk (Burkert \& Silk 2001), adiabatic SMBH growth (MacMillan \& Henriksen 2002), and direct gas collapse (Adams et al. 2001, 2003).

\subsection{Implications for Evolution in the $M_{B H}-\sigma$ Relation}

The existence of these two complementary $M_{B H}-\sigma$ relations also has potential consequences for measurements of the evolution of this correlation. Studies of AGN at intermediate redshift have led to somewhat ambiguous conclusions: some show evolution in $M_{B H}-\sigma$ (Treu et al. 2004, 2007; Walter et al. 2004; Woo et al. 2006, 2008), while others do not (Shields et al. 2003). Measurements of SMBH masses at intermediate redshift are done using reverberation mapping techniques (Blandford \& McKee 1982; Peterson 1993), which use spectral variability to infer the size scale of the broad line region and - assuming circular orbits - estimate the mass of the SMBH (see also Wandel et al. 1999; Kaspi et al. 2000b). These virial relations are calibrated using a relatively small sample of local, relatively lowluminosity AGN (Gebhardt et al. 2000b; Ferrarese et al. 2001; Onken et al. 2004; Nelson et al. 2004), many of which may reside in pseudobulge hosts and therefore calibrating them as classical bulges may not be appropriate.

However, at cosmological distances, AGN will be preferentially more luminous, and therefore more likely to be fueled by mergers than disk instabilities. This could lead to inferred evolution in the $M_{B H}-\sigma$ relation which is more related to selection effects than true evolution in the correlation. In fact, the shift of $\Delta \log \sigma \approx-0.15$ observed by Treu et al. (2004, 2007) and Woo et al. (2006, 2008 ) is entirely consistent simply with the difference between the normalizations of the merger and disk instability $M_{B H}-\sigma$ relations in Figure 12 .

\subsection{Why the Bulge?}

Both observational and theoretical work has demonstrated that the SMBH is correlated with the structural properties of its host galaxy. However, these correlations are far more significant when applied to the spheroidal 
component - or bulge - rather than the galaxy as a whole. We illustrate this in Figure 15, in which we show the binding energy correlation including all the stellar particles $\left(E_{\text {bind,all }}\right.$; left $)$, and the residual SMBH mass at fixed $E_{\text {bind,all }}$ as a function fo $E_{\text {bind,all }} / E_{\text {bind,b }}$ on the right, where $E_{\text {bind,b }}$ is an estimate of the total bulge binding energy assuming a Hernquist (1990) profile. When we use the correlation for major mergers, there is a clear trend in the residuals $-\mathrm{a} \sim 5 \sigma$ significant correlation towards an overprediction of the final SMBH mass when the bulge mass is small; $E_{\text {bind,all }}$ is not a good predictor of the SMBH for systems with significant disk contribution to the overall binding energy as compared to $E_{\text {bind,bulge. }}$

The question then arises of why, even in minor merger remnants where it contributes a small fraction of the total mass of the remnant, does the SMBH scale so tightly with the binding energy of the spheroidal component of the potential. There are two possible scenarios that may explain this correlation: (1) the bulge confines the gas inflow isotropically so that the SMBH can continue to accrete without ejecting gas along potential minima, or (2) the gas content of the final starburst dominates the local potential near the SMBH and therefore it grows until feedback is sufficient to unbind this local gas supply and terminate the inflow. While we defer a more thorough analysis of these two effects to future work, here we present some suggestive evidence for the latter over the former.

In Figure 16 we present the SMBH scaling with two different binding energy measures: that of the stellar component (the "stellar binding energy") after the remnant has relaxed, and that of the gaseous component (the "gas binding energy") at the peak of the final starburst. We furthermore restrict ourselves to the major merger series, for which it is a fair approximation that the remnant - including both the stars formed during the simulation and initialized with the progenitor disks - is entirely bulge dominated and largely gas free. We find that the relationship between the SMBH mass and the gaseous binding energy is somewhat steeper than that with the stellar binding energy: the logarithmic slopes are $\alpha=0.85 \pm 0.17$ and $0.74 \pm 0.13$ respectively. Moreover, this scaling with gas binding energy is statistically consistent with a linear relationship. This suggests - albeit tentatively - that (2) is more likely the dominant process. However, since the slope is not quite linear, it also argues for some contribution from the confinement of gas by the spheroidal potential.

\section{CONCLUSIONS}

We present a series of simulations of the self-regulated growth of SMBHs in which Eddington-limited accretion continues until a critical accretion rate, at which point feedback is sufficient to terminate the gas inflow and cut off further growth. Simulations of three different fuel- ing mechanisms - minor mergers, major mergers, and disk instabilities - all follow the same BHFP and binding energy correlations observed locally (Hopkins et al. 2007a; Aller \& Richstone 2007). Increasing the gas content of the initial disks shifts them along these correlations owing to structural changes in the resulting bulge arising from the effects of gas dissipation, but does not change their character. And, while the major and minor mergers both follow the same projected correlations - the

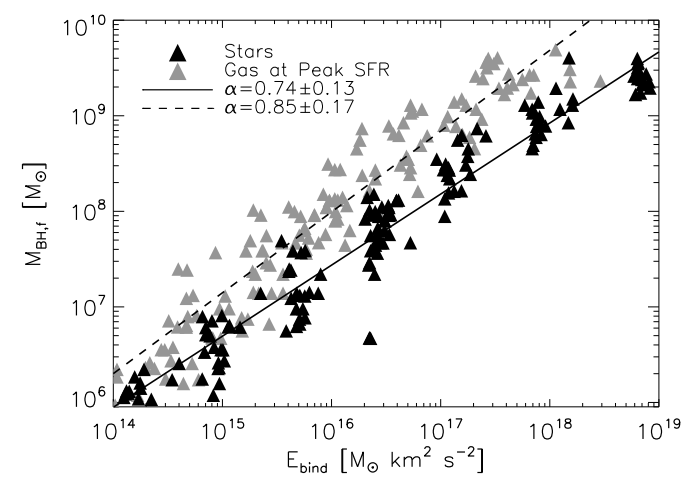

FIG. 16.- From the major mergers series: a comparison of the scaling of the final SMBH mass with the binding energy of the stellar bulge particles after the remnant reaches a state of approximate dynamical equilibrium (black triangles) and gas particles at the peak of the starburst (grey triangles), along with power-law fits to each (solid and dashed lines respectively). We find that the slope of the gas binding energy is steeper than that of the stellar particles, indicating that the relationship between SMBH mass and bulge binding energy reflects the SMBH self-regulating its growth by terminating the gas inflow, and that gas forming the bulge stars that dominate the potential close to the SMBH.

$M_{B H}-\sigma$ and Magorrian et al. (1998) relations - the unstable disks lie along different correlations, in agreement with observations of pseudobulges and bulges in barred systems (Hu 2008; Graham 2008), again reflecting the structural differences between bulges formed via mergers and secular processes (see also Kormendy \& Kennicutt 2004a). Taken together, these simulations support the $\mathrm{BHFP}$ as the most "fundamental" scaling relation, in that it reflects the physical mechanism driving the coevolution of SMBHs and bulges.

Thanks in particular to the anonymous referee for their helpful comments on this manuscript. We also thank Marijn Franx, Dusan Keres, Stephanie Bush, Gurtina Besla, Chris Hayward, Nick Scoville, Dave Sanders, Lisa Kewley, and Doug Richstone for helpful comments and discussions. These simulations were performed at the Harvard Institute for Theory and Computation. This work is supported in part by a grant from the W. M. Keck Foundation.

\section{REFERENCES}

Abadi, M. G., Navarro, J. F., \& Steinmetz, M. 2006, MNRAS, 365,747

Adams, F. C., Graff, D. S., Mbonye, M., \& Richstone, D. O. 2003, ApJ, 591, 125

Adams, F. C., Graff, D. S., \& Richstone, D. O. 2001, ApJ, 551, L31

Aller, M. C. \& Richstone, D. O. 2007, ApJ, 665, 120
Arav, N. et al. 2001, ApJ, 546, 140

Athanassoula, E. 1992, MNRAS, 259, 345

Athanassoula, E. 2000, in Astronomical Society of the Pacific Conference Series, Vol. 221, Stars, Gas and Dust in Galaxies: Exploring the Links, ed. D. Alloin, K. Olsen, \& G. Galaz, $243-+$

Ayal, S. \& Piran, T. 2001, ApJ, 555, 23 
Barnes, J. \& Hut, P. 1986, Nature, 324, 446

Barnes, J. E. 1992, ApJ, 393, 484

Barnes, J. E. \& Hernquist, L. 1992, ARA\&A, 30, 705

-. 1996, ApJ, 471, 115

Barnes, J. E. \& Hernquist, L. E. 1991, ApJ, 370, L65

Barway, S. \& Kembhavi, A. 2007, ApJ, 662, L67

Bell, E. F. \& de Jong, R. S. 2000, MNRAS, 312, 497

Benson, A. J. 2005, MNRAS, 358, 551

Berczik, P., Merritt, D., Spurzem, R., \& Bischof, H.-P. 2006, ApJ, 642, L21

Bett, P., Eke, V., Frenk, C. S., Jenkins, A., Helly, J., \& Navarro, J. 2007, MNRAS, 376, 215

Binney, J. \& Tremaine, S. 1987, Galactic dynamics (Princeton,

NJ, Princeton University Press, 1987, 747 p.)

Blandford, R. D. \& McKee, C. F. 1982, ApJ, 255, 419

Bondi, H. 1952, MNRAS, 112, 195

Bondi, H. \& Hoyle, F. 1944, MNRAS, 104, 273

Bournaud, F., Combes, F., \& Semelin, B. 2005, MNRAS, 364, L18

Bower, R. G., Benson, A. J., Malbon, R., Helly, J. C., Frenk, C. S., Baugh, C. M., Cole, S., \& Lacey, C. G. 2006, MNRAS, 370,645

Bullock, J. S. \& Johnston, K. V. 2005, ApJ, 635, 931

Bullock, J. S., Kolatt, T. S., Sigad, Y., Somerville, R. S., Kravtsov, A. V., Klypin, A. A., Primack, J. R., \& Dekel, A. 2001, MNRAS, 321, 559

Burkert, A. \& Silk, J. 2001, ApJ, 554, L151

Busha, M. T., Evrard, A. E., Adams, F. C., \& Wechsler, R. H. 2005, MNRAS, 363, L11

Cole, S. \& Lacey, C. 1996, MNRAS, 281, 716

Cox, T. J., Dutta, S. N., Di Matteo, T., Hernquist, L., Hopkins, P. F., Robertson, B., \& Springel, V. 2006, ApJ, 650, 791

Cox, T. J., Jonsson, P., Somerville, R. S., Primack, J. R., \& Dekel, A. 2008, MNRAS, 384, 386

Crenshaw, D. M., Kraemer, S. B., Boggess, A., Maran, S. P. Mushotzky, R. F., \& Wu, C.-C. 1999, ApJ, 516, 750

Crenshaw, D. M., Kraemer, S. B., \& George, I. M. 2003, ARA\&A, 41, 117

Croton, D. J. et al. 2006, MNRAS, 365, 11

Debattista, V. P., Mayer, L., Carollo, C. M., Moore, B., Wadsley, J., \& Quinn, T. 2006, ApJ, 645, 209

Di Matteo, T., Colberg, J., Springel, V., Hernquist, L., \& Sijacki, D. 2008, ApJ, 676, 33

Di Matteo, T., Springel, V., \& Hernquist, L. 2005, Nature, 433, 604

Djorgovski, S. \& Davis, M. 1987, ApJ, 313, 59

Dressler, A., Lynden-Bell, D., Burstein, D., Davies, R. L., Faber, S. M., Terlevich, R., \& Wegner, G. 1987, ApJ, 313, 42

Edgar, R. 2004, New Astronomy Review, 48, 843

Efstathiou, G., Lake, G., \& Negroponte, J. 1982, MNRAS, 199, 1069

Erb, D. K., Steidel, C. C., Shapley, A. E., Pettini, M., Reddy, N. A., \& Adelberger, K. L. 2006, ApJ, 646, 107

Escala, A., Larson, R. B., Coppi, P. S., \& Mardones, D. 2004, ApJ, 607, 765

Eskridge, P. B. et al. 2000, AJ, 119, 536

Faber, S. M. \& Jackson, R. E. 1976, ApJ, 204, 668

Ferrarese, L. \& Merritt, D. 2000, ApJ, 539, L9

Ferrarese, L. et al. 2001, ApJ, 555, L79

Förster Schreiber, N. M. et al. 2006, ApJ, 645, 1062

Gebhardt, K. et al. 2000a, ApJ, 539, L13

-. 2000b, ApJ, 543, L5

Geha, M., Blanton, M. R., Masjedi, M., \& West, A. A. 2006, ApJ, 653,240

Gingold, R. A. \& Monaghan, J. J. 1977, MNRAS, 181, 375

Graham, A. W. 2008, ApJ, submitted [astro-ph/0801.1548], 801

Graham, A. W. \& Driver, S. P. 2007, ApJ, 655, 77

Graham, A. W., Erwin, P., Caon, N., \& Trujillo, I. 2001, ApJ, 563, L11

Häring, N. \& Rix, H.-W. 2004, ApJ, 604, L89

Hernquist, L. 1989, Nature, 340, 687

-. 1990, ApJ, 356, 359

-. 1992, ApJ, 400, 460

-. 1993, ApJ, 409, 548

Hernquist, L. \& Katz, N. 1989, ApJS, 70, 419

Hernquist, L. \& Mihos, J. C. 1995, ApJ, 448, 41

Hernquist, L., Spergel, D. N., \& Heyl, J. S. 1993, ApJ, 416, 415

Hohl, F. 1971, ApJ, 168, 343
Hopkins, P. F., Cox, T. J., Kereš, D., \& Hernquist, L. 2008a, ApJS, 175, 390

Hopkins, P. F., Cox, T. J., Younger, J. D., \& Hernquist, L. 2008b, ApJ, submitted [astro-ph/0806.1739]

Hopkins, P. F. \& Hernquist, L. 2006, ApJS, 166, 1

Hopkins, P. F., Hernquist, L., Cox, T. J., Di Matteo, T., Martini, P., Robertson, B., \& Springel, V. 2005a, ApJ, 630, 705

Hopkins, P. F., Hernquist, L., Cox, T. J., Di Matteo, T.,

Robertson, B., \& Springel, V. 2005b, ApJ, 630, 716

-. 2005c, ApJ, 632, 81

-. 2006a, ApJS, 163, 1

Hopkins, P. F., Hernquist, L., Cox, T. J., Dutta, S. N., \& Rothberg, B. 2008c, ApJ, in press [astro-ph/0802.0508], 802

Hopkins, P. F., Hernquist, L., Cox, T. J., \& Kereš, D. 2008d, ApJS, 175, 356

Hopkins, P. F., Hernquist, L., Cox, T. J., Robertson, B., Di Matteo, T., \& Springel, V. 2006b, ApJ, 639, 700

Hopkins, P. F., Hernquist, L., Cox, T. J., Robertson, B., \& Krause, E. 2007a, ApJ, 669, 45

- 2007b, ApJ, 669, 67

Hopkins, P. F., Hernquist, L., Cox, T. J., Robertson, B., \& Springel, V. 2006c, ApJS, 163, 50

Hopkins, P. F., Hernquist, L., Martini, P., Cox, T. J., Robertson, B., Di Matteo, T., \& Springel, V. 2005d, ApJ, 625, L71

Hopkins, P. F. et al. 2008e, ApJ, submitted

-. 2008f, ApJ, submitted

—. 2008g, ApJ, submitted

-. 2008h, ApJ, submitted

Hoyle, F. \& Lyttleton, R. A. 1941, MNRAS, 101, 227

Hozumi, S. \& Hernquist, L. 2005, PASJ, 57, 719

$\mathrm{Hu}$, J. 2008, MNRAS, submitted [astro-ph/0801.1481], 801

Jogee, S., Kenney, J. D. P., \& Smith, B. J. 1999, ApJ, 526, 665

Jogee, S., Scoville, N., \& Kenney, J. D. P. 2005, ApJ, 630, 837

Jogee, S. et al. 2002, ApJ, 575, 156

-. 2004, ApJ, 615, L105

Johansson, P. H., Naab, T., \& Burkert, A. 2008, ApJ, submitted [astro-ph/0802.0210], 802

Kaastra, J. S., Mewe, R., Liedahl, D. A., Komossa, S., \&

Brinkman, A. C. 2000, A\&A, 354, L83

Kaastra, J. S. et al. 2002, A\&A, 386, 427

Kaspi, S., Brandt, W. N., Netzer, H., Sambruna, R., Chartas, G., Garmire, G. P., \& Nousek, J. A. 2000a, ApJ, 535, L17

Kaspi, S., Smith, P. S., Netzer, H., Maoz, D., Jannuzi, B. T., \& Giveon, U. 2000b, ApJ, 533, 631

Kaspi, S. et al. 2002, ApJ, 574, 643

Katz, N., Weinberg, D. H., \& Hernquist, L. 1996, ApJS, 105, 19

Kazantzidis, S., Bullock, J. S., Zentner, A. R., Kravtsov, A. V., \&

Moustakas, L. A. 2007, ApJ, submitted (astro-ph/0708.1949)

Kennicutt, Jr., R. C. 1998, ApJ, 498, 541

Khochfar, S. \& Burkert, A. 2006, A\&A, 445, 403

King, A. 2003, ApJ, 596, L27

-. 2005, ApJ, 635, L121

Korista, K. T., Voit, G. M., Morris, S. L., \& Weymann, R. J. 1993, ApJS, 88, 357

Kormendy, J. 2004, in Coevolution of Black Holes and Galaxies, ed. L. C. Ho, 1-+

Kormendy, J. \& Kennicutt, Jr., R. C. 2004a, ARA\&A, 42, 603

—. 2004b, ARA\&A, 42, 603

Kormendy, J. \& Richstone, D. 1995, ARA\&A, 33, 581

Kriss, G. A. et al. 2000, ApJ, 538, L17

Lucy, L. B. 1977, AJ, 82, 1013

Macciò, A. V., Dutton, A. A., van den Bosch, F. C., Moore, B., Potter, D., \& Stadel, J. 2007, MNRAS, 378, 55

MacMillan, J. D. \& Henriksen, R. N. 2002, ApJ, 569, 83

Magorrian, J., Tremaine, S., Richstone, D., Bender, R., Bower G., Dressler, A., Faber, S. M., Gebhardt, K., Green, R. Grillmair, C., Kormendy, J., \& Lauer, T. 1998, AJ, 115, 2285

Makino, J. \& Funato, Y. 2004, ApJ, 602, 93

Marconi, A. \& Hunt, L. K. 2003, ApJ, 589, L21

McLure, R. J. \& Dunlop, J. S. 2002, MNRAS, 331, 795

Menéndez-Delmestre, K., Sheth, K., Schinnerer, E., Jarrett, T. H., \& Scoville, N. Z. 2007, ApJ, 657, 790

Mihos, J. C. \& Hernquist, L. 1994, ApJ, 431, L9

—. 1996, ApJ, 464, 641

Milosavljević, M. \& Merritt, D. 2001, ApJ, 563, 34

Miralda-Escudé, J. \& Kollmeier, J. A. 2005, ApJ, 619, 30 
Murray, N., Quataert, E., \& Thompson, T. A. 2005, ApJ, 618, 569

Navarro, J. F., Frenk, C. S., \& White, S. D. M. 1996, ApJ, 462, 563

Nelson, C. H., Green, R. F., Bower, G., Gebhardt, K., \& Weistrop, D. 2004, ApJ, 615, 652

Novak, G. S., Faber, S. M., \& Dekel, A. 2006, ApJ, 637, 96

Onken, C. A. et al. 2004, ApJ, 615, 645

Ostriker, J. P. \& Peebles, P. J. E. 1973, ApJ, 186, 467

Peterson, B. M. 1993, PASP, 105, 247

Petitpas, G. R. \& Wilson, C. D. 2002, ApJ, 575, 814

—. 2003, ApJ, 587, 649

Quinn, P. J. \& Goodman, J. 1986, ApJ, 309, 472

Quinn, P. J., Hernquist, L., \& Fullagar, D. P. 1993, ApJ, 403, 74

Roberts, M. S. \& Haynes, M. P. 1994, ARA\&A, 32, 115

Robertson, B., Bullock, J. S., Cox, T. J., Di Matteo, T., Hernquist, L., Springel, V., \& Yoshida, N. 2006a, ApJ, 645, 986

Robertson, B., Cox, T. J., Hernquist, L., Franx, M., Hopkins, P. F., Martini, P., \& Springel, V. 2006b, ApJ, 641, 21

Robertson, B., Hernquist, L., Cox, T. J., Di Matteo, T., Hopkins, P. F., Martini, P., \& Springel, V. 2006c, ApJ, 641, 90

Robertson, B., Yoshida, N., Springel, V., \& Hernquist, L. 2004, ApJ, 606, 32

Ruderman, M. A. \& Spiegel, E. A. 1971, ApJ, 165, 1

Sazonov, S. Y., Ostriker, J. P., Ciotti, L., \& Sunyaev, R. A. 2005, MNRAS, 358, 168

Schmidt, M. 1959, ApJ, 129, 243

Schombert, J. M., McGaugh, S. S., \& Eder, J. A. 2001, AJ, 121, 2420

Sersic, J. L. 1968, Atlas de galaxias australes (Cordoba, Argentina: Observatorio Astronomico, 1968)

Shen, J. \& Sellwood, J. A. 2004, ApJ, 604, 614

Sheth, K. et al. 2008, ApJ, 675, 1141

Shields, G. A. et al. 2003, ApJ, 583, 124

Sijacki, D., Springel, V., di Matteo, T., \& Hernquist, L. 2007, MNRAS, 380, 877

Silk, J. \& Rees, M. J. 1998, A\&A, 331, L1
Soltan, A. 1982, MNRAS, 200, 115

Springel, V. 2005, MNRAS, 364, 1105

Springel, V., Di Matteo, T., \& Hernquist, L. 2005, MNRAS, 361, 776

Springel, V. \& Hernquist, L. 2002, MNRAS, 333, 649

-. 2003, MNRAS, 339, 289

-. 2005, ApJ, 622, L9

Stewart, K. R., Bullock, J. S., Wechsler, R. H., Maller, A. H., \&

Zentner, A. R. 2007, ApJ, in press [astro-ph/0711.5027], 711

Thacker, R. J., Scannapieco, E., \& Couchman, H. M. P. 2006, ApJ, 653, 86

Toomre, A. 1964, ApJ, 139, 1217

Toomre, A. 1981, in Structure and Evolution of Normal Galaxies, ed. S. M. Fall \& D. Lynden-Bell, 111-136

Tremaine, S. et al. 2002, ApJ, 574, 740

Treu, T., Malkan, M. A., \& Blandford, R. D. 2004, ApJ, 615, L97

Treu, T., Woo, J.-H., Malkan, M. A., \& Blandford, R. D. 2007, ApJ, 667, 117

Velazquez, H. \& White, S. D. M. 1999, MNRAS, 304, 254

Vitvitska, M., Klypin, A. A., Kravtsov, A. V., Wechsler, R. H., Primack, J. R., \& Bullock, J. S. 2002, ApJ, 581, 799

Walker, I. R., Mihos, J. C., \& Hernquist, L. 1996, ApJ, 460, 121

Walter, F. et al. 2004, ApJ, 615, L17

Wandel, A., Peterson, B. M., \& Malkan, M. A. 1999, ApJ, 526, 579

Weymann, R. J., Morris, S. L., Foltz, C. B., \& Hewett, P. C. 1991, ApJ, 373, 23

Woo, J. ., Treu, T., Malkan, M. A., \& Blandford, R. 2008, ApJ, in press [astro-ph/0804.0235]

Woo, J.-H., Treu, T., Malkan, M. A., \& Blandford, R. D. 2006, ApJ, 645, 900

Younger, J. D., Cox, H. T. J., Seth, A. C., \& Hernquist, L. 2007, ApJ, 670, 269

Zhao, H., Haehnelt, M. G., \& Rees, M. J. 2002, New Astronomy, 7, 385 